\title{
Suitability of NB-IoT for Indoor Industrial Environment: A Survey and Insights
}

\author{
Muhammad Dangana*(D), Shuja Ansari (D), Qammer H. Abbasi (D, Sajjad Hussain (D) and Muhammad Ali Imran (D)
}

Citation: Dangana, M.; Ansari, S.; Abbasi, Q.H.; Hussain, S.; Imran, M.A. Suitability of NB-IoT for Indoor Industrial Environment: A Survey and Insights. Sensors 2021, 21, 5284. https://doi.org/10.3390/s21165284

Academic Editor: Pietro Manzoni

Received: 16 June 2021

Accepted: 28 July 2021

Published: 5 August 2021

Publisher's Note: MDPI stays neutral with regard to jurisdictional claims in published maps and institutional affiliations.

Copyright: (c) 2021 by the authors. Licensee MDPI, Basel, Switzerland. This article is an open access article distributed under the terms and conditions of the Creative Commons Attribution (CC BY) license (https:// creativecommons.org/licenses/by/ $4.0 /)$.
James Watt School of Engineering, University of Glasgow, Glasgow G12 8QQ, UK; Shuja.Ansari@glasgow.ac.uk (S.A.); Qammer.Abbasi@glasgow.ac.uk (Q.H.A.); Sajjad.Hussain@glasgow.ac.uk (S.H.); Muhammad.Imran@glasgow.ac.uk (M.A.I.)

* Correspondence: m.dangana.1@research.gla.ac.uk

\begin{abstract}
The Internet of Things (IoT) and its applications in industrial settings are set to bring in the fourth industrial revolution. The industrial environment consisting of high profile manufacturing plants and a variety of equipment is inherently characterized by high reflectiveness, causing significant multi-path components that affect the propagation of wireless communications-a challenge among others that needs to be resolved. This paper provides a detailed insight into Narrow-Band IoT (NB-IoT), Industrial IoT (IIoT), and Wireless Sensor Networks (WSN) within the context of indoor industrial environments. It presents the applications of NB-IoT for industrial settings, such as the challenges associated with these applications. Furthermore, future research directions were put forth in the areas of NB-IoT network management using self-organizing network (SON) technology, edge computing for scalability enhancement, security in NB-IoT generated data, and proposing a suitable propagation model for reliable wireless communications.
\end{abstract}

Keywords: Narrow-Band IoT; Internet of Things; Industrial IoT; wireless communication; latency; self-organizing network (SON); edge computing; long term evolution (LTE)

\section{Introduction}

The development of ad hoc Wireless Sensor Networks (WSN) contributes to the paradigm of the Internet of Things (IoT). Many wireless technologies have emerged with this paradigm; among these are the Low Power Wide Area Network (LPWAN) such as Narrow-Band IoT (NB-IoT), Long Term Evolution for Machines (LTE-M), Long Range Wide Area Network (LoRaWAN), SigFox, etc. Since IoT involves the connectivity of massive objects to the internet for information or data gathering, monitoring, and control, a highly reliable platform is required. As such, the focus has been on the requirements that will establish this reliable connectivity. Wireless technologies are therefore geared towards meeting these requirements among which include: low power consumption, to enable longer duration of connectivity and energy savings; coverage range, for extended object reachability; reliability, for error-free data transmission and network availability; security, for data confidentiality, integrity, and availability; latency, for real-time data delivery.

The standardization of NB-IoT technology by the Third Generation Partnership Project (3GPP) in 2016 [1] has empowered NB-IoT with features that have enabled it to be suitable for wireless data communication. The expanded Discontinuous Reception (eDRx) and Power Saving Mode (PSM) techniques allow NB-IoT to implement its low power consumption scheme. For a more extended coverage range, the technology employs the re-transmission and low-frequency modulation mechanisms. For a low latency sensitivity level, 3GPP has prescribed a tolerable latency level of 10s. The superimposition of NB-IoT on Long Term Evolution (LTE) provides the support and reliability needed for its network availability.

These 3GPP standardization features have increased the presence of NB-IoT modules in the forecast of the IoT device market share by 2030 [2]. Many wireless vendors have 
entered into the business of IoT manufacturing. Since NB-IoT is integrated into the LTE infrastructure, this has resulted in its application in numerous industrial sectors, such as agriculture, transportation, automobile factories, logistics and manufacturing, while increasing the data transmission over LTE infrastructure. However, the structural settings of these industries differ and are comparatively unique. The uniqueness of these industries is characterized by heavy machinery and in some cases with metals that are highly reflective to wireless signals. These forms of settings present a harsh condition for wireless communications [3].

The deployment of NB-IoT in indoor industrial environments is faced with these harsh channel conditions, and as such, a more appropriate wireless propagation model is needed to describe the wireless system of NB-IoT communication in an indoor industrial environment. Attempts have been made to characterize the wireless propagation of narrowband in industrial settings [3,4]. Reference [3] performed physical measurements of wood and metal processing factories for large-scale and temporal fading at frequencies of 900 , 2400, and $5200 \mathrm{MHz}$. The authors identified and classified the features of the industrial environments that affect the large-scale and temporal fading. Of interest is the presentation made by [4] on its comparative analysis of indoor industrial environment propagation models with a generic representation of path-loss for $900 \mathrm{MHz}$.

This paper presents a survey on NB-IoT, and as a future direction, some research areas are discussed in a bid to bring forth an appropriate representation of the NB-IoT network. This is to aid in designing suitable wireless communication systems that can be used in developing a reliable scheme for the measurement of an acceptable threshold for NB-IoT wireless performance in industries, measuring the tolerable latency sensitivity level as described in 3GPP, and analyzing the effect of the propagation model on power consumption rates in NB-IoT.

After the introduction section, in this paper, Section 2 presents the technical background on NB-IoT with brief explanations on WSN, IoT, and IIoT; this is to clarify their relations and impact on NB-IoT. Other topics presented include the standardization and technology of NB-IoT. As shown in the organizational structure of the research article in Figure 1, under the state-of-the-art, Section 3.1 discusses the developmental trends and technical features of NB-IoT, WSN, and IoT. Section 3.2 discusses the industrial application case studies of NB-IoT. The challenges posed by the industrial environments on these technologies are presented in Section 3.3. Table 1 also shows a summary of selected state-of-the-art articles. Further research directions and conclusions are presented in Sections 4 and 5, respectively.

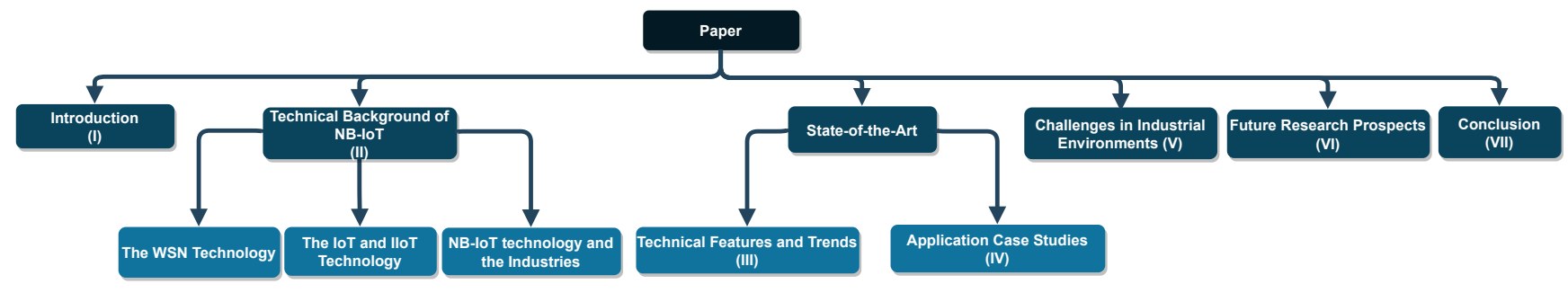

Figure 1. A structural description of the paper layout. 
Table 1. Highlights of some research work.

\begin{tabular}{|c|c|c|c|}
\hline Category & Discussion & Ref & Year \\
\hline \multirow{14}{*}{ NB-IoT } & Survey the features of NB-IoT and its application in industries & [5] & 2019 \\
\hline & Features of some LPWAN are compared for industrial applications & [6] & 2019 \\
\hline & Overview of NB-IoT & {$[1,7]$} & 2017 \\
\hline & Evaluates the coverage performance of NB-IoT & [8] & 2016 \\
\hline & Presents NB-IoT architecture through the perspective of 3GPP & [9] & 2017 \\
\hline & Explanations on the automation of Industries with Narrow-Band Internet of Things Technology & [10] & 2020 \\
\hline & Did a comparative analysis between two LPWAN viz; NB-IoT and LoRa on Power Wireless Private Network (PWPN) & [11] & 2018 \\
\hline & Evaluates the Power Saving Mode (PSM) and Expanded Discontinues Reception (eDRx) schemes of NB-IoT and a developed energy model & [13] & 2018 \\
\hline & Surveyed the energy efficiency of NB-IoT, its applications and challenges & [14] & 2019 \\
\hline & Highlights the applications and implementations of NB-IoT & [15] & 2017 \\
\hline & NB-IoT, SigFox, Lora and GPRS coverage range were compared in an area of $7800 \mathrm{~km}^{2}$ & [16] & 2017 \\
\hline & A study of the technical representation of NB-IoT from physical to its media access control layers & [17] & 2019 \\
\hline & Proposed an uplink adaptation scheme for uplink scheduling in NB-IoT & [18] & 2017 \\
\hline & Studied the performance of NB-IoT and eMTC in the implementation of smart cities & [19] & 2018 \\
\hline \multirow{5}{*}{ IIoT } & Analytically proposes IIoT architecture & [20] & 2018 \\
\hline & Review on IIoT & {$[22,23]$} & 2018 \\
\hline & Optimized QoS through wireless transmission in IIoT & [24] & 2020 \\
\hline & Demonstration of IIoT by the inclusion of gateway & [25] & 2019 \\
\hline & Uses machine learning for IIoT & [26] & 2020 \\
\hline \multirow{3}{*}{ IoT } & Identify the evolution of radio frequency identification in IoT technology & [27] & 2008 \\
\hline & Survey the impact of IoT on technology, social, and businesses & [28] & 2018 \\
\hline & Survey the architecture and features of IoT & [29-34] & $\begin{array}{l}2018,2017,2016, \\
2020,2019,2019\end{array}$ \\
\hline \multirow{7}{*}{ WSN } & WSN and its types surveyed & [35] & 2018 \\
\hline & Survey WSN in Agriculture & [36] & 2016 \\
\hline & Discuss the application of WSN in the monitoring of the environment & [37] & 2012 \\
\hline & Presents the requirements and challenges in the application of WSN in oil and gas industries & [38] & 2018 \\
\hline & Proposes the use of WSN and IoT in oil and gas industries & [39] & 2017 \\
\hline & Described application of WSN in industrial automation & [40] & 2008 \\
\hline & The standardization process in industrial WSN for industrial automation & [41] & 2010 \\
\hline
\end{tabular}




\section{Research Methodology}

Among the contributions of this paper are, firstly, to review the state-of-the-art research in the field of NB-IoT technology and its applications in industrial environments. To review other IoT technologies (WSN, IoT, and IIoT) upon which NB-IoT is established. This is aimed at understanding the relationships and impact of these technologies with NB-IoT, especially in terms of technical features, applications, and challenges in industrial environments. Lastly, to highlight the challenges and future directions of wireless communication of NB-IoT in industries. This paper reviewed research carried out in recent years in the field of Industrial Internet of Things (IIoT) application with some notes on earlier contributions. The process of this review involved a thorough checking of the significant contributions made by journals, articles, conference papers, and report papers. The scholarly databases were systematically queried to extract important information from the literature identified. A total of 368 papers were pulled out of the databases, and 116 papers were selected and comprehensively read, analyzed, and categorized based on their areas of review. Figure 2 depicts the paper extraction procedures. The first process screened out or excluded papers that were in duplicates, non-English written, and had text wrongfully categorized concerning the area of review interest. The review process began with the inclusion of papers on the basis of titles, abstracts, keywords, and conclusions. A total number of 116 papers were finally selected by article type (survey or review, theoretical, practical, etc.). Data were also extracted at this stage to include both theoretical and experimental contents. The third process of the review focused on the challenges that are related to the communication characteristics as supported by the network layers.

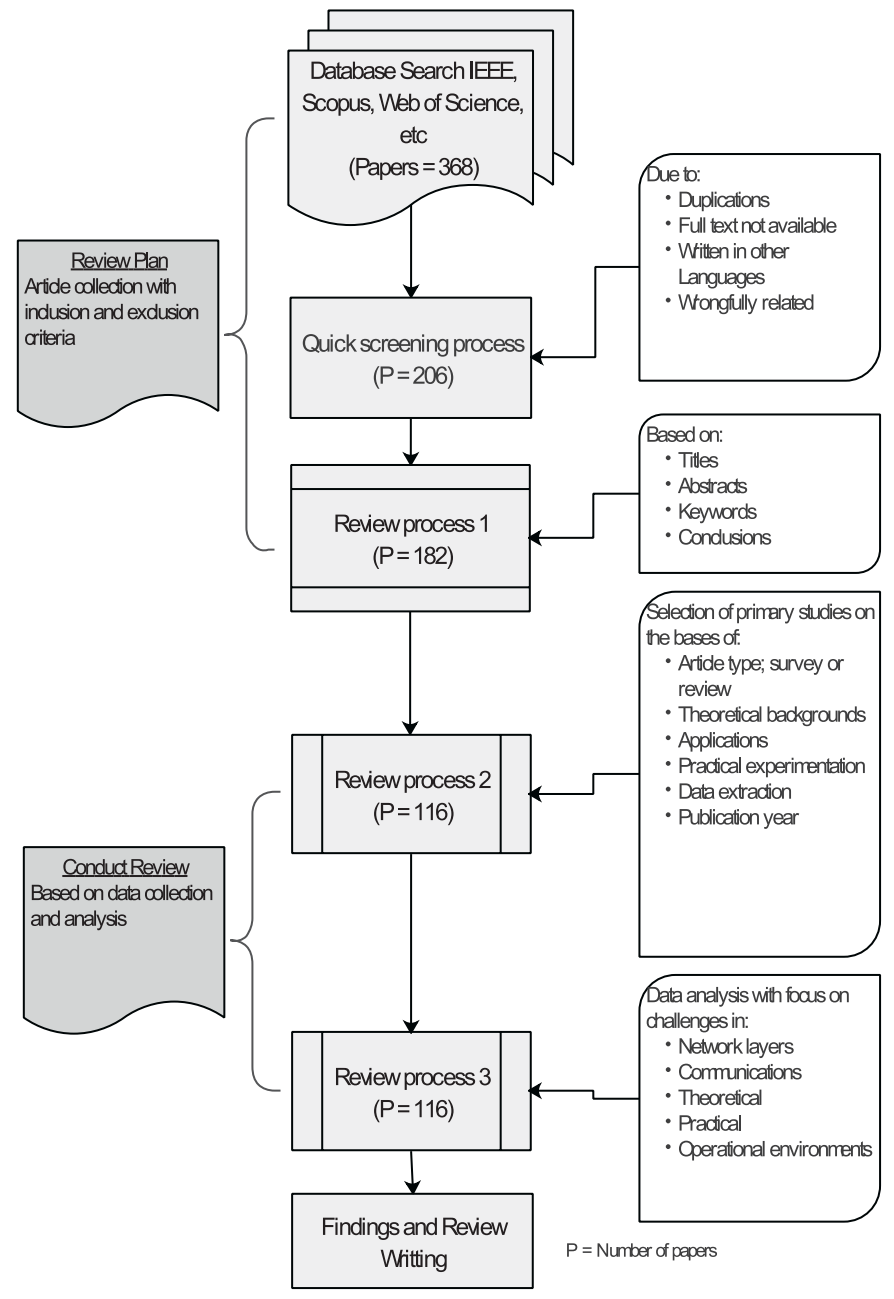

Figure 2. Data extraction procedure. 


\section{Technical Background of NB-IoT}

The evolution of the Internet of Things (IoT) has occurred in recent years, with a forecast on IoT device connectivity running into billions in 2021 [2]. The standardization and careful research into technology has also led to meaningful progress. This section, therefore, briefly describes some of the features of WSN, IoT, Industrial IoT (IIoT), and NB-IoT, as well as their technical challenges.

\subsection{The Wireless Sensor Networks (WSN) Technology}

WSN is a set of sensing nodes that are arranged in a manner that allows them to function together to monitor or read some physical quantity (such as temperature, humidity, pressure, etc.) and communicate to a central point or device wirelessly. The full structure of WSN comprises the sensors (nodes), the communication technique, and the nature of their inter-connectivity. This implies that WSN has the capabilities of sensing, processing, storing, and communicating with its data destination through the wireless medium.

\subsubsection{Structure of a Typical Sensor Node}

To understand the WSN technology, a study of a typical sensor node is important. Figure 3 shows the four major units of a node: power, sensing, processing, and transceiver units. The power unit provides the energy required by other units in the node. This power source in most cases is provided by a battery. The sensing unit monitors the physical quantities that are presented in analog form or signals. With the aid of an Analog-to-Digital converter (ADC), the analog signal is converted to a digital signal. Both the sensor and $\mathrm{ADC}$ form the sensing unit. The digital signal is then processed by a micro-controller or processor. The processed data are stored temporarily in the storage section of the processing unit. The transceiver unit enables the node to connect with other nodes or the gateway in the network [42].

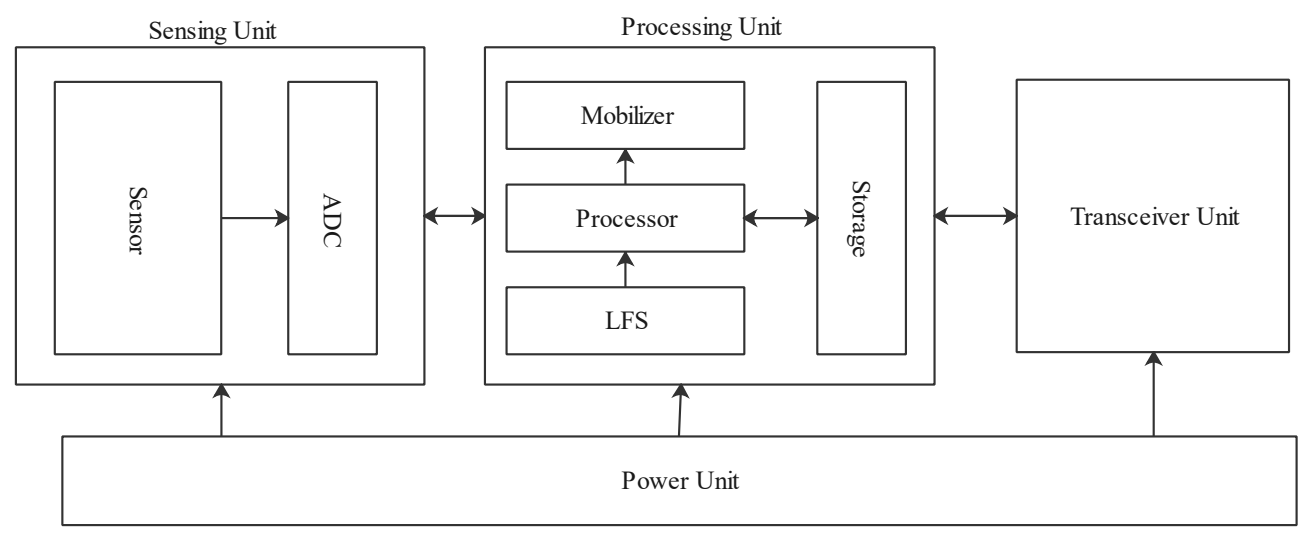

Figure 3. A detailed representation of the four units of an IoT node.

\subsubsection{WSN Network Architecture}

The network architecture of WSN is considered to be of two types: structured and unstructured [43]. A structured WSN network design provides for a well-mannered approach to its planning and distribution of the nodes. With this type of deployment, the network maintenance rate is minimal, which leads to a low management expenditure as well. While in an unstructured network, the nodes' distribution and placements are scattered without proper planning. This type of design features dense and random deployment of nodes. This will lead to uncovered areas of deployment in the field, which may in-turn need monitoring. Since the nodes are not carefully placed, node failure detection and connectivity management become difficult to handle. Sensor nodes are of different types, which are designed or described based on the type of physical quantity they monitor. As shown in Figure 4, sensors such as humidity, temperature, flow, vibration, power sensors, etc., are connected wirelessly to a base station that is capable of aggregating data gathered from the 
sensors to the network server. The user computer connects with the server to access the data and further process the data, which will be presented in a format that is more understandable by the decision making team. The wireless media used by these nodes enables a multi-hop communication. These communication links could be provided by radio or infrared technologies. Where a radio link is used, WSN often uses the unlicensed frequency band known as the Industrial, Scientific, and Medical (ISM) band [42]. These frequency bands are specified by the International Table of Frequency Allocations, and they also provide huge spectrum allocation and are unregulated but highly prone to interference.

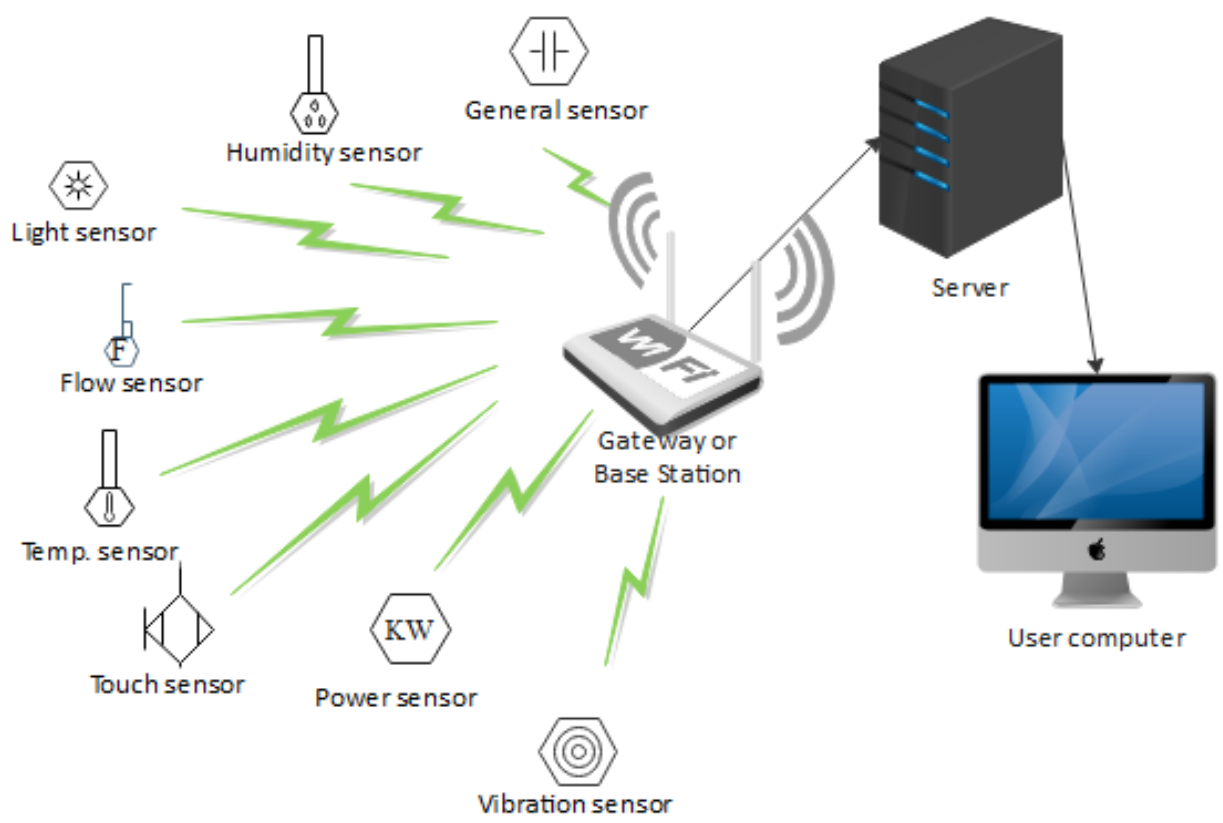

Figure 4. A WSN network architecture showing data sources, aggregation, storage, and control points.

\subsubsection{WSN Topology and Types}

The description of WSN topology is associated with the expected capabilities of sensor nodes, which enable the deployment of several numbers of nodes in a sensor field [44]. While four major types of topologies can be adapted for field deployment, such as, star, tree, point-to-point, and mesh topologies [36], well-planned handling of these arrangements are necessary for maintenance purpose [42]. Without a proper topology arrangement, maintenance-related issues become difficult to handle. These issues usually arise in deployment phases: pre-deployment and deployment phase, post-deployment phase, and redeployment of more nodes phase [42]. In some cases, the types of WSN are classified based on the field of application, such as health, industry, military, agriculture, etc. [45]. However, WSN types can be identified as terrestrial, underground, underwater, mobile, and multimedia wireless sensor networks [46].

\subsubsection{Challenges Facing WSN}

Although WSN technology has had performance improvements over the years [47], with many research questions getting resolved, other challenges are still present. Some of these challenges arise from node structure, while others are posed by the environment in which they operate in. Reference [48] highlighted some of these design challenges encountered by WSN. These include the operational environments, where WSN are deployed in areas that affect its operation, such as the industries, underwater, underground, etc.; the power consumed by nodes to process its data, transmit it, or receive any data; the scalability of these nodes, since they are deployed in large numbers for a specific application; 
provision of good quality of service; fault tolerance in the network; limitation in computational and storage capabilities; security of data in terms of confidentiality, integrity, and availability.

\subsection{The Internet of Things (IoT) and Industrial Internet of Things (IIoT) Technologies}

The advancement in IoT has lessened direct human intervention with objects that are capable of sharing information among themselves and the environment. Designed with sensing and actuating capabilities, these objects have become smart, considering their ability to communicate and autonomously react to real-time or physical events through a unifying infrastructure $[49,50]$. The interactions of sensors with Radio Frequency Identification (RFID) and the introduction of Internet Protocol (IP), which enables the identification of nodes and their location as well as easy routing on the Internet [51], have transformed WSNs into IoT. Figure 5 shows the forecasted increase in the IoT devices by 2030, however, this is an estimate as signifies by the asterisk. As the number of IoT devices grows tremendously to about 50 billion worldwide [2], the IPv4 identification process will be exhausted, and IPv6 becomes useful for supporting this increase in the IoT paradigm.

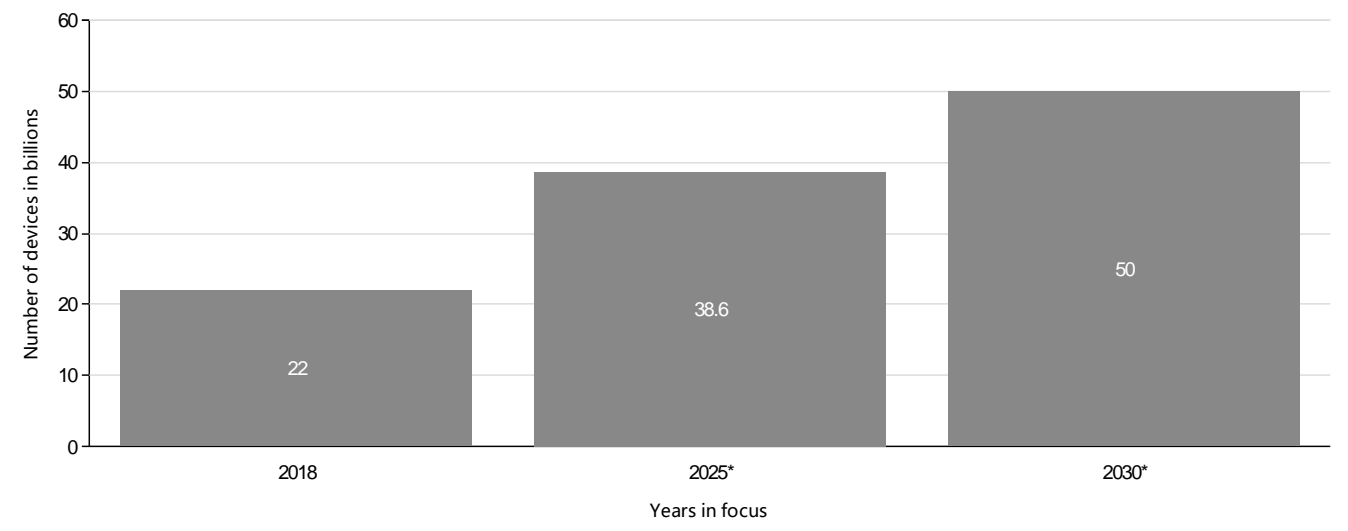

Figure 5. Forecast of the number of IoT-connected devices by 2030 [2].

Industrial IoT is simply the introduction or application of IoT in industries, especially in manufacturing $[20,50]$. IoT connectivity is mostly consumer-grade and is used for building automation, human wearables, and messaging, etc., while in IIoT, the connectivity is secured and targeted at the automation of industrial processes such as in defense, aerospace industries, etc. [50]. Table 2 further shows the comparisons between IoT and IIoT based on their respective characteristics [21].

Table 2. Comparisons of IoT and IIoT characteristics.

\begin{tabular}{cccccc}
\hline Types & Data Volume & Connectivity & $\begin{array}{c}\text { Exchange of } \\
\text { Information }\end{array}$ & Market Segment & Criticality \\
\hline IoT & Big data & $\begin{array}{c}\text { Consumer grade, e.g., } \\
\text { smart homes, } \\
\text { entertainment, etc. } \\
\text { Business to consumers, } \\
\text { business to business to } \\
\text { consumers }\end{array}$ & $\begin{array}{c}\text { Service providers, } \\
\text { consumers, limited } \\
\text { enterprises, and } \\
\text { small businesses }\end{array}$ & $\begin{array}{c}\text { Not stringent } \\
\text { (Excluding medical } \\
\text { applications) }\end{array}$ & Revolution \\
\hline IIoT & $\begin{array}{c}\text { Specific and } \\
\text { limited data }\end{array}$ & $\begin{array}{c}\text { Secured, e.g., health care, } \\
\text { energy, etc. }\end{array}$ & $\begin{array}{c}\text { Business to business } \\
\text { only }\end{array}$ & $\begin{array}{c}\text { Enterprises and } \\
\text { Industries }\end{array}$ & $\begin{array}{c}\text { Critical for } \\
\text { timing, reliability, } \\
\text { privacy, and } \\
\text { security }\end{array}$ \\
\hline
\end{tabular}




\subsubsection{IoT the Enabling Technology for IIoT}

Cloud computing, Machine-to-Machine (M2M), Artificial Intelligent (AI), and Blockchain are among the large group of technologies that enables effective and reliable IIoT [52], with IoT forming the backbone. Figure 6 shows the structure of a typical IoT technology. The things here are regular objects, such as a VR headset, watch, electric cooker, parking lot or garage, security cameras, shopping cart, etc., that are made smart because of the sensor nodes embedded in them. This sensing ability enables these things to interact through a common infrastructure, the Internet, with a management center for decision making. The interaction is possible through the various wireless communication technologies available. For the protection of data, a firewall is designed to secure the information exchange between the objects and the center. Communication device manufacturers have provided solutions to protect this data. The network layer provides the platform on which different communication technologies can function. These include long-range licensed and unlicensed wireless bands. The licensed wireless technology, such as 2G/3G and LTE involves, the use of existing telecommunication infrastructures, while the unlicensed wireless (LoRa, ZigBee, etc.) is an ad hoc setup with Internet capability (through an Internet modem or a gateway). The smart objects, set up in this fashion, have various applications such as in homes, city environment, traffic, etc. Their management is also done through any internet-empowered device such as smartphones, laptops, tablets, etc. Figure 7 represents the application of IoT in the industrial environment, the IIoT. In industrial applications, the sensor nodes are embedded in the industrial equipment for monitoring, operations, and management. The communication technologies remain the same; however, the processes that are managed are of critical nature. Applications include oil and gas industries, chemical, automobile, food processing industries, etc. While IoT could be less stringent in terms of criticality, IIoT is critical with timing, reliability, privacy, and security of data being gathered in operation or monitoring.

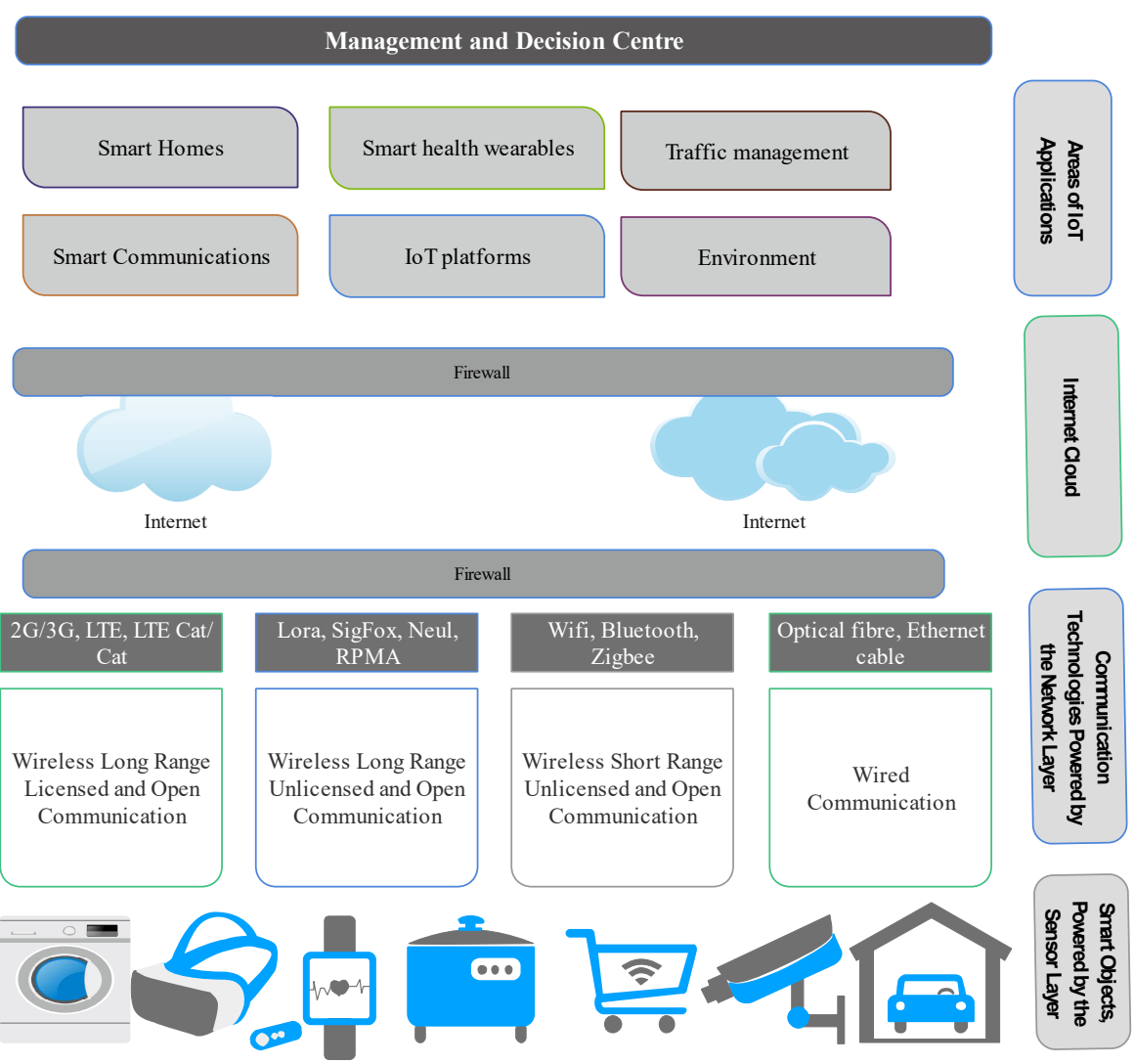

Figure 6. A typical structure of consumer-based IoT showing basic layers. 


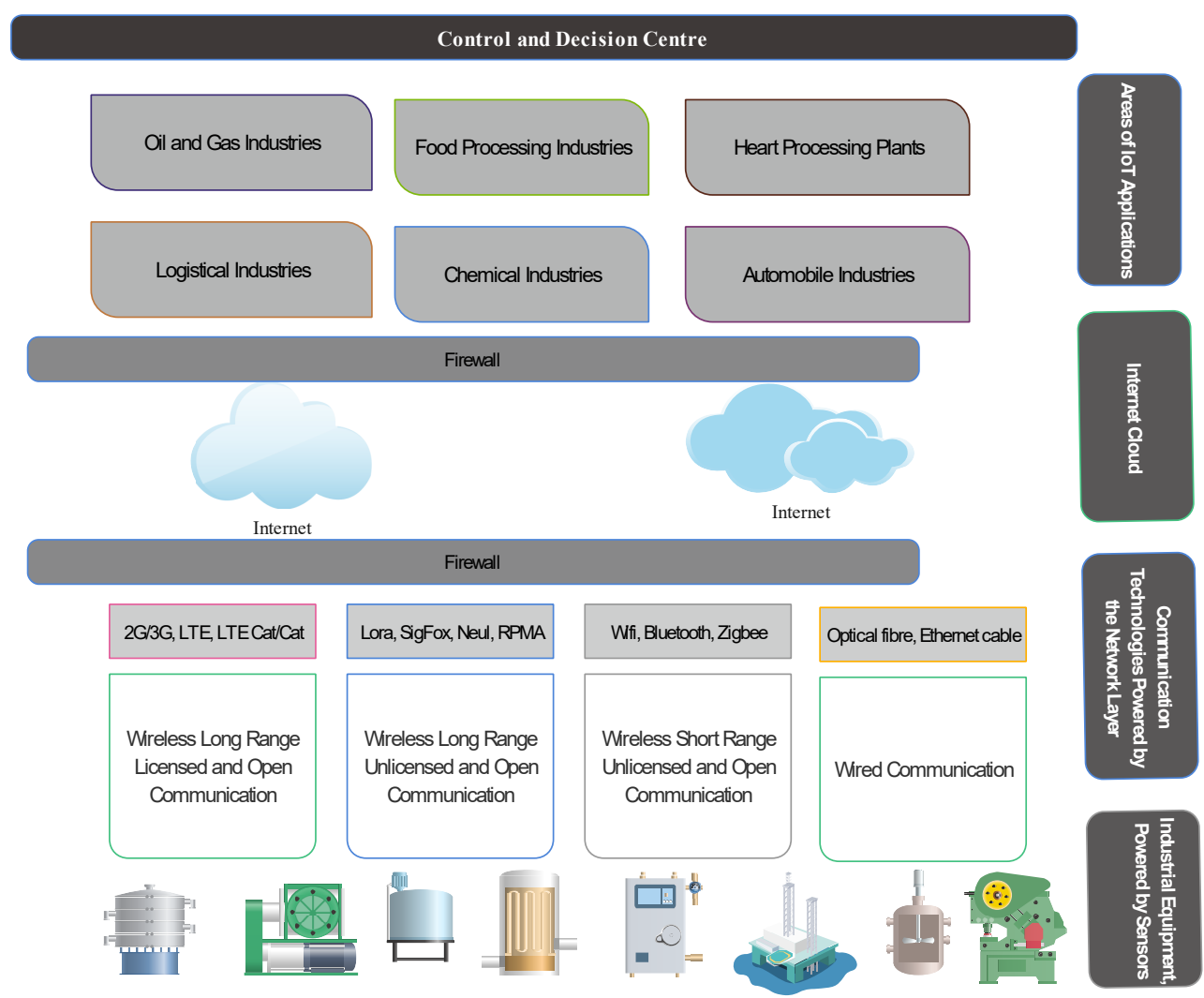

Figure 7. A typical structure of Industrial IoT (IIoT) showing basic layers.

\subsubsection{The IoT/IIoT Communication Technologies}

IoT and IIoT are thought to have three or five architectural functionality layers [53,54]. Meanwhile, in [55], for a Man Like Nervous (MLN) system, other layers are involved. Threelayered IoT/IIoT architecture include perception, network, and application layers. While in five-layered architecture, in addition to the three layers are processing and business layers. However, in any type of architecture proposed or adopted, the network or transport [53] layer is responsible for the communication of devices among themselves and with the network infrastructure (e.g., gateway, server, etc.). IIoT allows the industries to meet their operational targets, among other things. However, most of the peculiar needs of the industries are centered on the capabilities of communication technologies as supported by the network layer. These designs need to include latency-time required for data propagation and processing, topology-device connection style, throughput-the volume of data that the network can support, scalability-number of supported devices in the network, the security of data and energy-IoT device operational time without power supply [56]. Table 3 lists the common types of communication technologies used by IoT/IIoT and the network-supported features.

The network or transport layer handles the coverage range of IoT/IIoT. Each type of communication technology has an approximate range it can cover. Therefore, depending on the requirements needed for an application, appropriate technology can be employed. However, for IIoT where timing is critical, latency is an important feature that needs to be put into consideration to achieve reasonable reliability of deployment. To this end, a true picture or understanding of the industrial wireless communication for the application of IoT is necessary. 
Table 3. Common IoT/IIoT wireless communication features.

\begin{tabular}{|c|c|c|c|c|c|c|c|c|}
\hline Type & Latency & Bandwidth & Data Rate & Coverage Range & Energy & Security & Scalability & Ref. \\
\hline NB-IoT & $1-10 \mathrm{~s}$ & $200 \mathrm{KHz}$ & $200 \mathrm{kbps}$ & $\begin{array}{c}1 \mathrm{~km} / 10 \mathrm{~km} \\
\text { (Urban/Rural) }\end{array}$ & 10 years battery life & Yes & 52,000 & {$[5,6,9,57-59]$} \\
\hline LoRaWAN & $600 \mathrm{~ms}$ & $\begin{array}{c}250 \mathrm{KHz} \text { and } \\
125 \mathrm{KHz}\end{array}$ & $50 \mathrm{kbps}$ & $\begin{array}{c}5 \mathrm{~km} / 20 \mathrm{~km} \\
\text { (Urban/Rural) }\end{array}$ & $>10$ years battery life & Yes & $\begin{array}{c}\text { Approx. } 104 \\
\text { nodes/BS }\end{array}$ & {$[5,6,58-60]$} \\
\hline SigFox & $1-20 \mathrm{~s}$ & $100 \mathrm{~Hz}$ & $100 \mathrm{bps}$ & $\begin{array}{c}10 \mathrm{~km} / 40 \mathrm{~km} \\
\text { (Urban/Rural) }\end{array}$ & $>10$ years battery life & Yes & $\begin{array}{c}\text { Approx. } 106 \\
\text { nodes/BS }\end{array}$ & {$[5,6,58-60]$} \\
\hline Bluetooth & $200-500 \mathrm{~ms}$ & $1 \mathrm{MHz}$ & $3 \mathrm{Mbps}$ & $10 \mathrm{~m}$ & 72 microwatts & Yes & 8 & {$[58,59,61-64]$} \\
\hline Wifi & $20-250 \mathrm{~ms}$ & $22 \mathrm{MHz}$ & $\begin{array}{l}11 \text { Mbps- } \\
10 \text { Gbps }\end{array}$ & $100 \mathrm{~m}$ & 0.2 watt & Yes & 2007 & {$[58,59,61-64]$} \\
\hline ZigBee & $60-150 \mathrm{~ms}$ & $\begin{array}{c}0.3 / 0.6 \mathrm{MHz} \\
2 \mathrm{MHz}\end{array}$ & $250 \mathrm{kbps}$ & $10-100 \mathrm{~m}$ & 90 microwatts & Yes & 65,000 & {$[58,59,61-63,65]$} \\
\hline
\end{tabular}

\subsubsection{IIoT Challenges}

Some of the challenges faced by Industrial IoT are the requirements needed for their deployment in various settings. Although different fields of application have their requirements [21], most of these requirements are expected or provided by the network layer of the IoT architecture. For example, in [66], the scalability, throughput, latency, energy consumption, topology, and security of data were explained. More so, the interoperability of devices to support the heterogeneous nature of IoT is presented as a future requirement for IoT in [67]. Standardization, architecture, and the need for an increase in storage are some other challenges that IIoT faces [68]. This research paper presents the challenges that are related to the network layer.

Scalability: The capacity provided by the global information infrastructure to connect a large number of common objects describes the scalability. This challenge can present itself on different levels [69]. The addressing and identification of devices pose a challenge; however, this issue is mitigated by the use of IPv6 and 6LoWPAN protocols [70]. Due to the large size of these connected objects, a high volume of data communication (big data) and networking capabilities are issues to be tackled. The application layer also contributes to the challenge posed by scalability as a result of the numerous services and service execution options that are generated by heterogeneous connected IoT devices. Therefore, one of the challenges in scalability is the ease of adding new devices to the network without major interruption.

Latency: Since the industrial application of IoT is time-critical and usually deployed in environments that are noisy and hard on the wireless communications, the Quality of Service (QoS) provided by IIoT is often characterized by the real-time achievement of the deadline set by an end-to-end communication task, from sensing to control execution in the system [71]. In industries, various communication traffic is generated, and depending on the type, the latency and reliability provided by IIoT can either be least critical or most critical. These types of traffic are explained in [72]. Table 4 presents a summary of the latency and reliability levels needed for the communication traffic.

Industrial traffic (data) that requires fractions of seconds in latency in IIoT communications includes the emergency, critical control, and remote control traffics. This traffic is used for safety purposes, such as the leakage of radiation or poisonous gases in industries [72], continual flow of industrial processes such as automation processes, which require $1 \mathrm{~ms}$ latency rate [73], and control of unmanned vehicles, which require $50 \mathrm{~ms}$ latency and $1-10^{-8}$ reliability [74].

Energy consumption: The primary energy supply to IoT is batteries. The replacement of these batteries becomes difficult when they are fully drained. However, energy harvesting techniques are becoming promising in resolving this challenge. Ideas and research are already moving toward techniques where solar panels or piezoelectric material will be used to relieve IoT devices from the shackles of battery operations [69]. Meanwhile, efforts have been made to optimize the network protocols in the wireless communication systems. These protocols are optimized to help in reducing the amount of energy consumed by these devices. Examples are the implementation of idle time of the devices when transmission or reception of data is not taking place. 
Security: Data generated by IIoT are important assets to the industry management, and while lack of proper security of these data is yet to be provided, this provides the reason why some industries are yet to fully deploy IoT. Although some traditional security mechanisms are in use, these are not sufficient enough to protect the complexity of the IIoT systems. These mechanisms include secure protocols in [75], privacy assurance in [76], and lightweight cryptography, as described in [77].

Table 4. Latency and reliability summary for industrial communication traffic.

\begin{tabular}{|c|c|c|c|c|}
\hline Traffic Types & Areas of Application & Reliability & Latency & Criticality \\
\hline Emergency traffic & Safety traffic & Ultra-high & Sub-milliseconds & Most \\
\hline Critical control traffic & Robotic motion control & Ultra-high & Milliseconds & \\
\hline Remote control traffic & Unmanned vehicle control & High & Milliseconds & \\
\hline Critical Alerting & Periodic control checks & Medium & Seconds & \\
\hline Non-critical Alerting & Temperature measurement & Low & Minutes & \\
\hline Monitoring & Static feedback & Low & Minutes & Least \\
\hline
\end{tabular}

\subsection{The Standardization and Technology of NB-IoT}

NB-IoT is one of the licensed frequency band communication technologies that were standardized by the 3GPP [1]. This technology is fused into LTE infrastructure as it is also an LPWAN-based technology. Among its category of LPWAN but utilizing the unlicensed frequency bands are LoRaWAN, Ingenu, SigFox, etc. NB-IoT is characterized by lowcost deployment and long-range coverage [17]. The use of the already existing cellular infrastructure by NB-IoT makes it a good candidate for the deployment of IoT as it provides a standardized common platform for the connectivity of objects. Among the advantages of NB-IoT include support for an effective cellular communication network, wide radius coverage with bidirectional triggering between data and signaling planes, low power consumption rate, and capacity to support the massive connections of devices $[15,78]$.

\subsubsection{Standardization of NB-IoT}

NB-IoT has passed through a series of standardization by 3GPP. Figure 8 describes a brief history of NB-IoT standardization from inception to its current freezing state. The diagram is a unique representation of the standardization history as it captures the various stages at a single glance. The standardization started in 2005 when 3GPP Release 8 (R8) was specified to cater for massive device connections, billing of usage, addressing, machine type communication mode, and security issues. All the standardization versions have a start date and end date with overlapping of some specific technological fields of concern. Examples are the versions R12 and R13 with various standardization numbers and specific areas of concern. In 2015, Version R13 was released with features that further reduced the energy consumption rate of NB-IoT through the implementation of eDRX and PSM mechanisms [15].

Figure 9 shows the major technology companies involved in the standardization process [79]. These companies include Vodafone and Huawei; they paired together to work on NB-IoT M2M communication features and made their submission in May 2014; Qualcomm presented NB-IoT Orthogonal Frequency-Division Multiplexing (OFDM) in August 2014. The works of the above formed the NB Cloud IoT (NB CIoT); a year later, Ericsson presented the LTE features of NB. These works were fused by rapporteurs in September 2015 to form the NB-IoT 3GPP Version R13 work item with a standardization number of 45.820. In this version, the following technological features were addressed: reduced latency, improved indoor coverage, ability to be compatible with existing infrastructure (LTE), support for a low data and low cost terminal. 


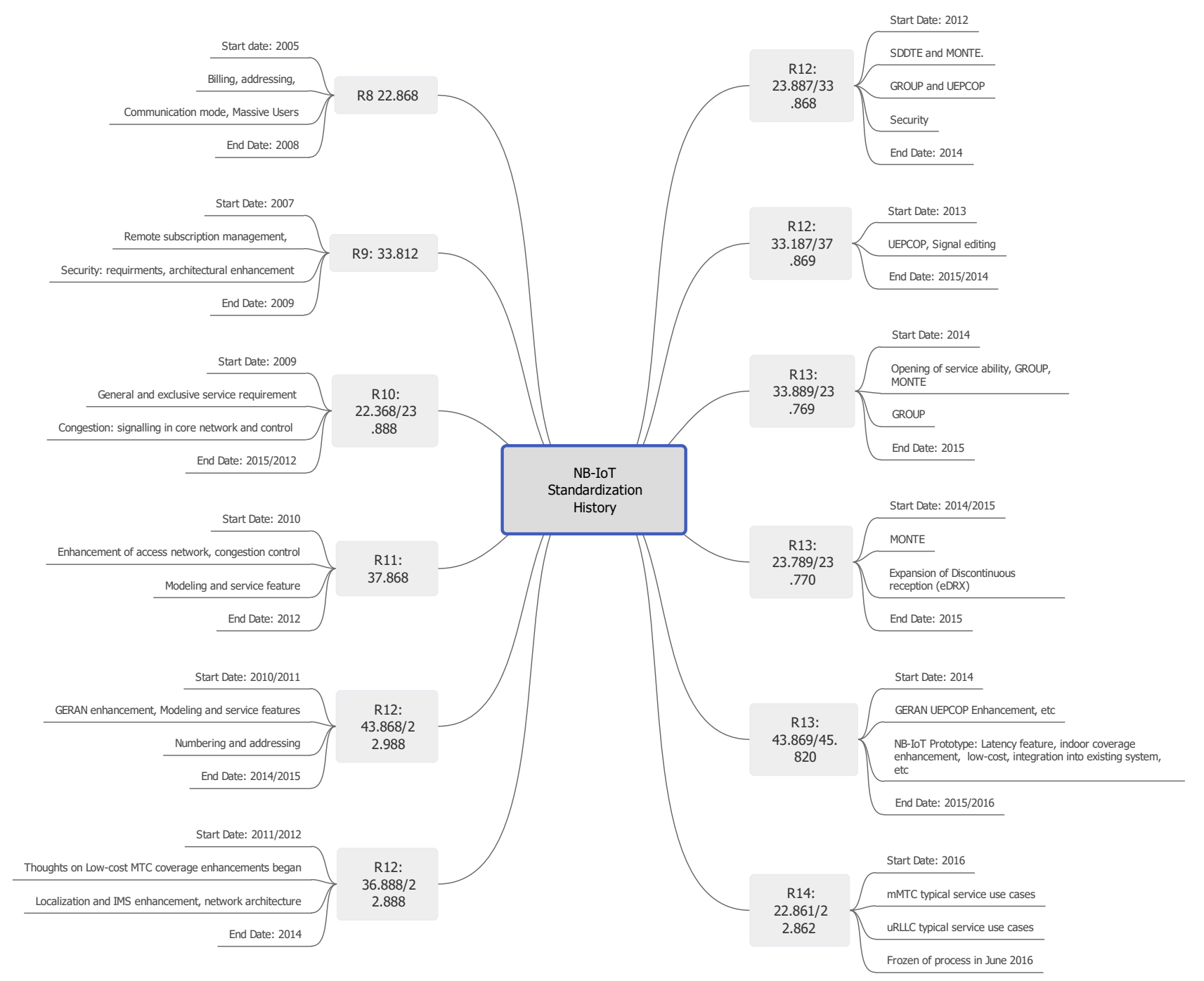

Figure 8. A unique representation of the NB-IoT standardization process.

\subsubsection{NB-IoT and Its Features}

Some of the main features of NB-IoT are enhanced coverage range, low energy consumption rate meaning long battery life, capacity to connect a massive number of devices per cell, increased reliability, low cost of the terminal and various deployment modes. These features are briefly explained below.

Coverage and Latency: NB-IoT gives better coverage as compared to the legacy LTE with $20 \mathrm{~dB}$ performance. This allows it to deliver to areas that are difficult to reach, such as basements, making it suitable for use in underground car parks. NB-IoT can achieve this coverage either by in-band, stand-alone, or guard band deployment modes. The $20 \mathrm{~dB}$ coverage enhancement is supported by retransmission and low-frequency modulation mechanisms. With the two retransmission tones ( 3.7 and $15 \mathrm{kHz}$ ) available for use, NB-IoT can retransmit using up to 128 bits for uplink and 2048 bits for downlink. The latency budget of NB-IoT is $10 \mathrm{~s}$, but a lower latency of $6 \mathrm{~s}$ can be achieved for maximal coupling losses as simulated and specified in TR45.820 [1,17]. 


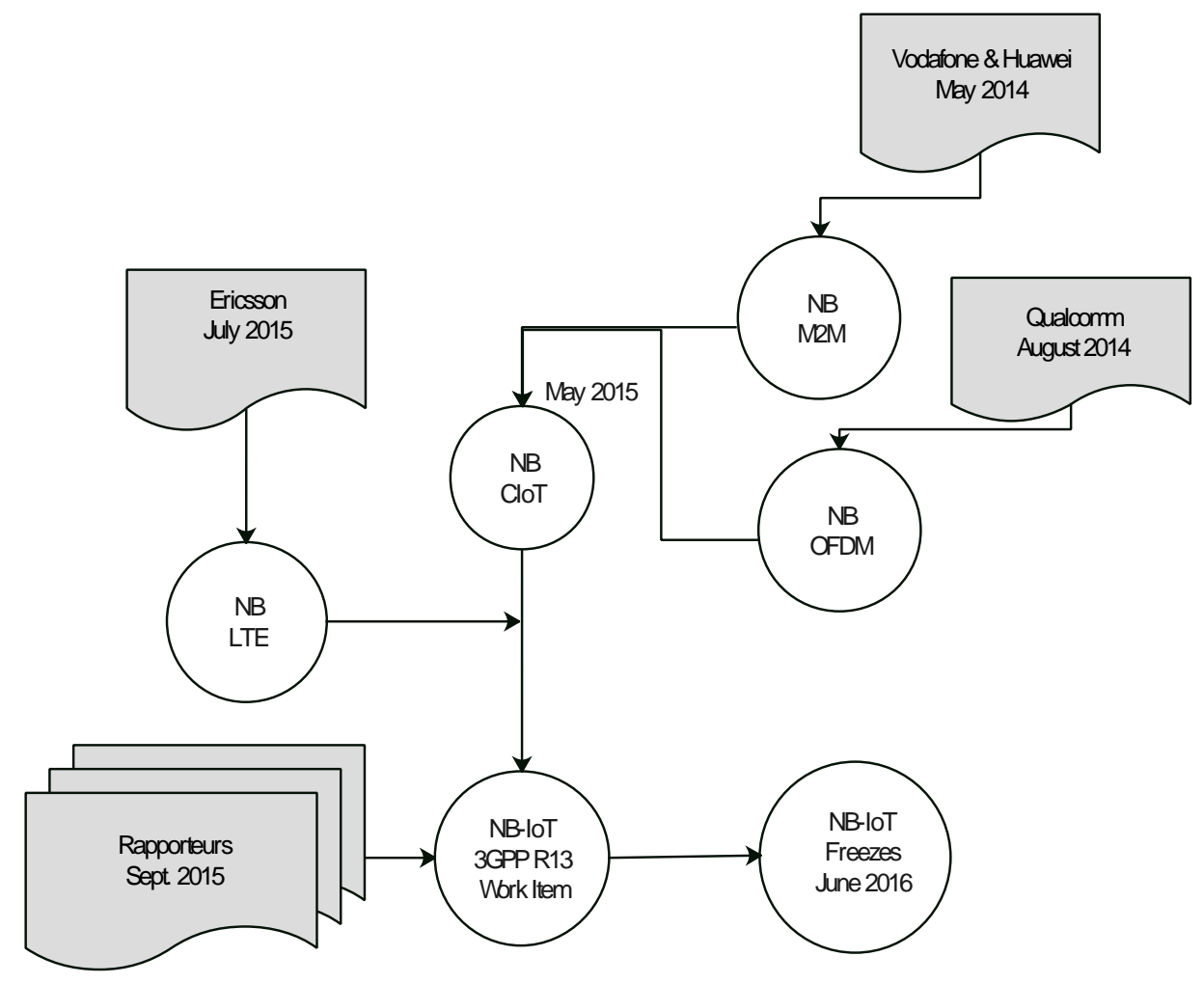

Figure 9. Participating companies in NB-IoT standardization.

The transmission tone feature, which enhances the coverage capability of NB-IoT is presented uniquely in a block diagram in Figure 10. The diagram presents the operational bandwidths of NB-IoT; $200 \mathrm{kHz}$ for stand-alone deployment and $180 \mathrm{kHz}$ for both inbound and guard-band deployments. For the uplink transmission, NB-IoT uses a Single Carrier Frequency-Division Multiplexing Access (SC-FDMA) modulation scheme. With this modulation scheme, it operates at 3.75 or $15 \mathrm{kHz}$ sub-carrier intervals. These singletone carriers are at 32 and $8 \mathrm{~ms}$, respectively. The transmission rates used are from 160 to $200 \mathrm{~kb} / \mathrm{s}$ to achieve a large coverage range that is powered by its high spectral density. The downlink transmission, however, uses an OFDMA modulation scheme with only a $15 \mathrm{kHz}$ sub-carrier interval. It also utilizes multi-tone carriers of 3, 6, and $12 \mathrm{~ms}$ that support 160 to $250 \mathrm{~kb} / \mathrm{s}$ transmission rates. The coverage enhancement of NB-IoT to achieve a $20 \mathrm{~dB}$ was evaluated in [80], and it was shown that this is true as compared to the legacy LTE.

Low energy consumption: The standardization process of the NB-IoT introduced the PSM and eDRX mechanisms for low energy consumption. In the PSM, the device remains registered to the network of transmission but usually enters a deep sleep mode and completely switches off most of its circuitry; at this stage, the device is not reachable from the network. Meanwhile, it could wake up at any time to transmit data when necessary. The eDRX is a temporary idle state that does not listen to the radio channel but periodically becomes active to receive paging messages from the network for possible incoming data before switching to PSM. This periodic activeness is guided by a specified timer, and the paging system is the process of monitoring the control channel for downlink or uplink data indication. Without any activity on the control channel and the expiration of the specified timer, the device switches from the idle state to the PSM state. These two basic features allow for the energy saving of NB-IoT, which surpasses the legacy LTE. Figure 11 shows the idle and paging states [13]. Timer T3324 is the time required to enter into deep sleep mode. 


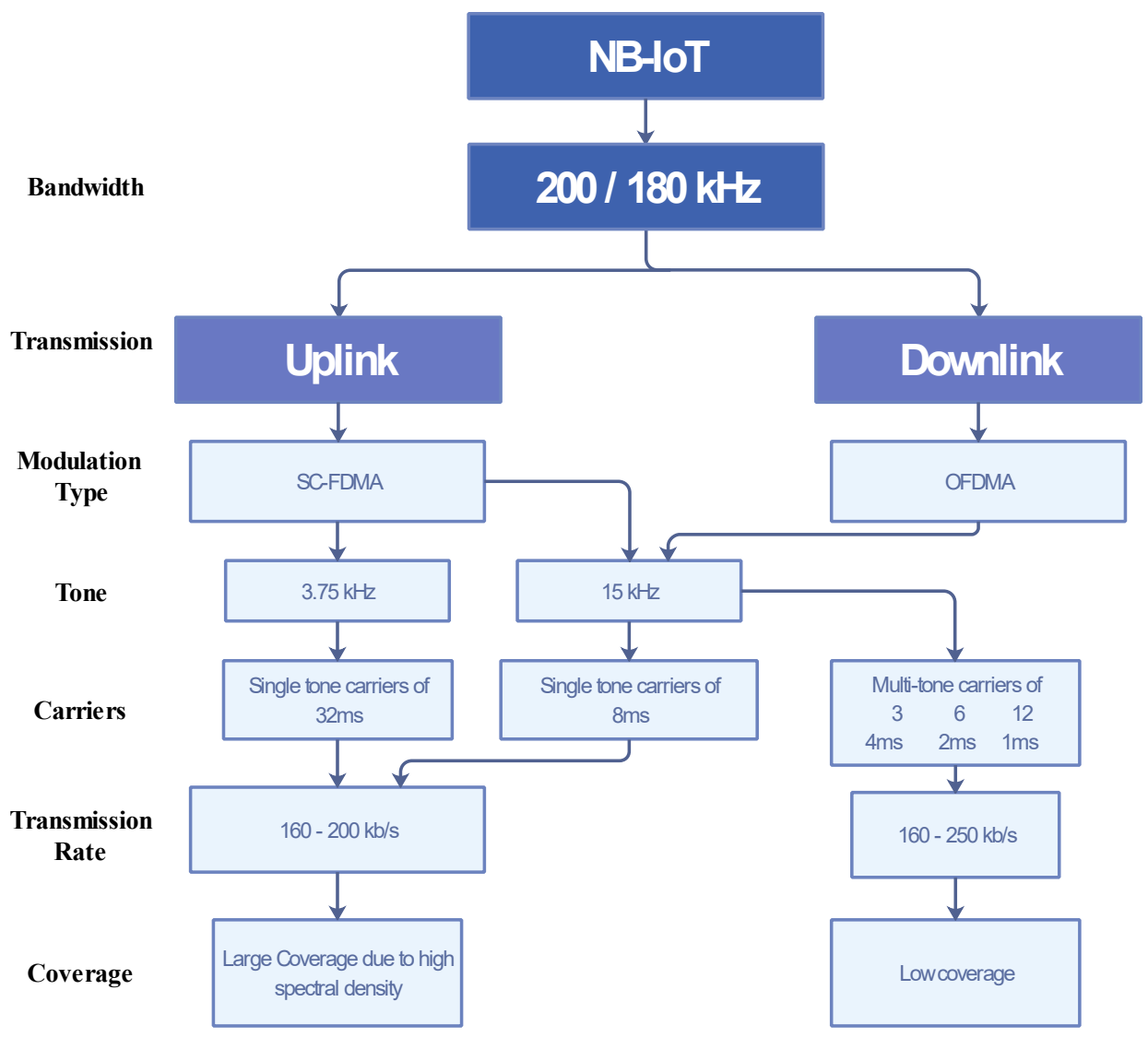

Figure 10. Block diagram of transmission tone mechanism.

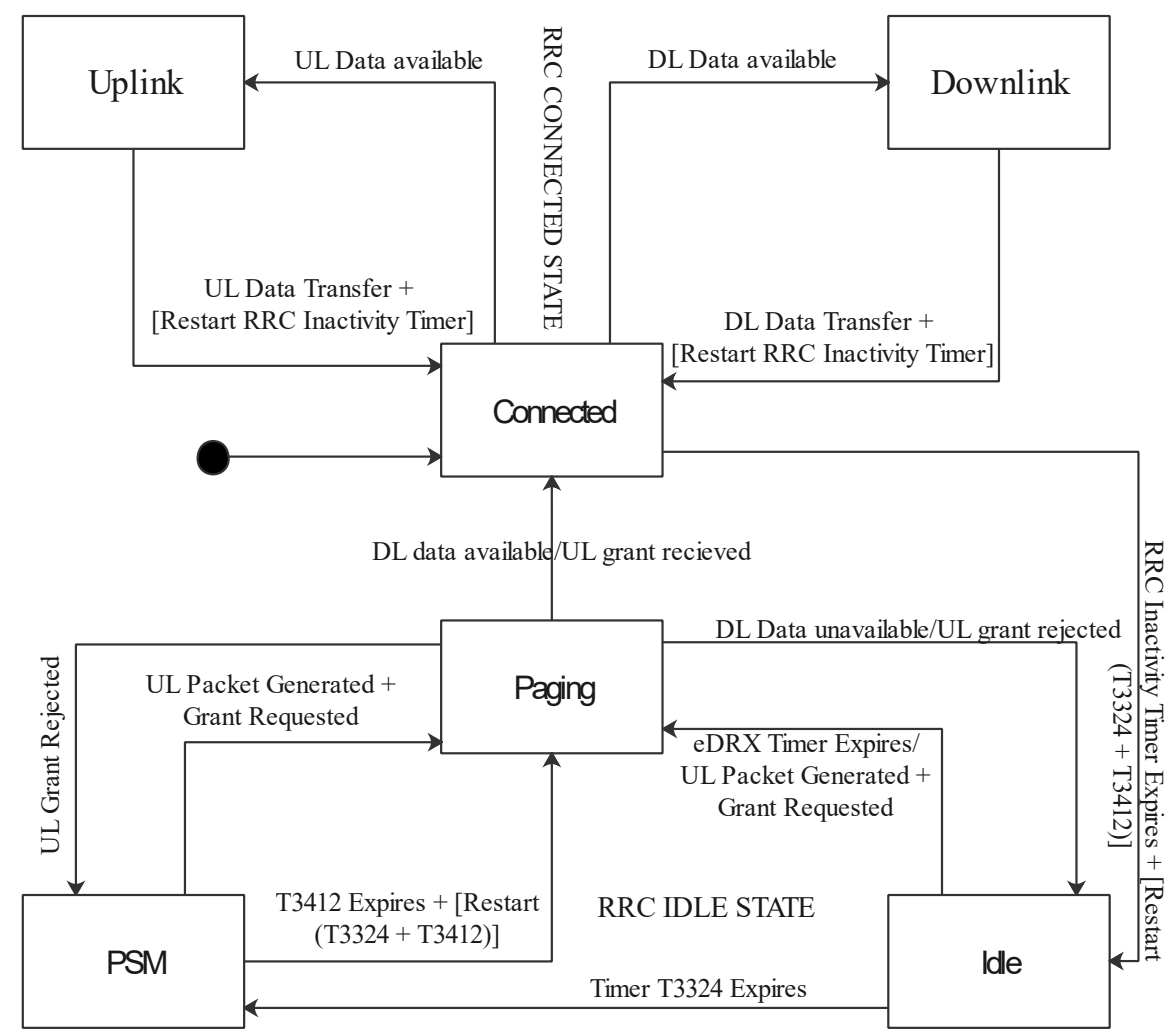

Figure 11. The PSM and eDRX modes. 
NB-IoT Deployment modes: NB-IoT is stipulated by RP-151621 to be deployed in FDD transmission [15] and has three types of deployment modes. These include the stand-alone, in-band, and guard-band modes. Figure 12 presents these modes. The stand-alone is an independent type of deployment that utilizes a bandwidth of $200 \mathrm{kHz}$ and does not overlap with the LTE frequency band. The guard-band is a deployment type that utilizes the guard frequency of the LTE or the edge band. It has a $180 \mathrm{kHz}$ bandwidth allocation. Lastly, the in-band deployment is also assigned a bandwidth of $180 \mathrm{kHz}$ and utilizes one of the LTE frequency bands.

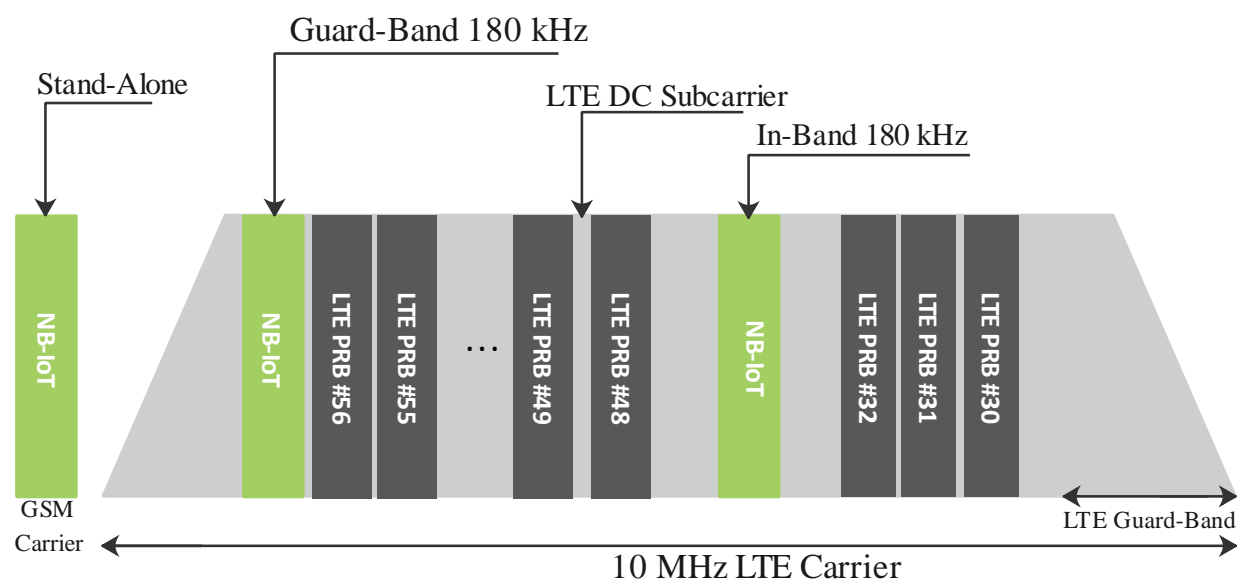

Figure 12. NB-IoT modes of deployment.

\subsubsection{NB-IoT Comparison with Other IoT Technologies}

In Table $5[1,7,16,18]$, the comparison between NB-IoT, other licensed, and unlicensed band IoT is presented. The IoT operating in the licensed bands include eMTC, while LoRaWAN, SigFox, Ingenu, etc., are for the unlicensed band.

Comparisons between NB-IoT and other LPWAN have been carried out to evaluate some features. These features include the cost of deployment, coverage range, interference immunity, power, and spectral efficiencies $[6,7,16,81,82]$. However, the results obtained showed that these technologies have some distinctive features that are suitable for specific purposes or applications. In some cases, trade-offs are required by the user for their deployments. An example is the deployment of NB-IoT in industries. Such decisions would leverage the existing infrastructure, security, and coverage of the LTE. Meanwhile, a decision that chooses LoRa, would require additional network infrastructure for deployment.

Table 5. Comparison between NB-IoT frequency band and other bands.

\begin{tabular}{ccccc}
\hline Parameters & eMTC & NB-IoT & LoRa & SigFox \\
\hline Spectral Bandwidth & $1.4 \mathrm{MHz}$ & $180 \mathrm{KHz}$ & $7.8-500 \mathrm{KHz}$ & $200 \mathrm{KHz}$ \\
& $700-900$ & $700-900$ & 868 & 868 \\
Spectral Frequency Band $(\mathrm{MHz})$ & Cellular & Cellular & ISM & ISM \\
& Licensed & Licensed & Unlicensed & Unlicensed \\
Spectral Efficiency & High & $<50 \mathrm{kbps}$ & Very Low & Very Low \\
Data Rate & $<1 \mathrm{Mbps}$ & $<15 \mathrm{~km}$ & $<10 \mathrm{kbps}$ & $100 \mathrm{bps}$ \\
Coverage Area & $<10 \mathrm{~km}$ & $2-3$ & $<10 \mathrm{~km}$ & $<12 \mathrm{~km}$ \\
Terminal Cost $(\$-2020)$ & 3.3 & Medium-High & Very High & 2.64 \\
Power Efficiency & Medium-High & Medium & Very High & High \\
Interference Immunity & Medium & LeRaWAN2 & Medium \\
Standard & 3GPP Release 14 & 3 GPP Release 13 & LTSI &
\end{tabular}




\subsubsection{NB-IoT Applications in Industries}

The application of NB-IIoT spans many of the industrial sectors with varying degrees of deployments [20]. This usually depends on the criticality and level of confidence in NB-IoT technology. However, its application has been forecast to increase in the foreseeable future especially with the increase in the manufacturing of NB-IoT modules by various vendors [2]. NB-IoT is deployed for different purposes in industries, as shown in Figure 13. In the oil and gas industries, NB-IoT deployment includes refining, distribution, and monitoring of products. Applications are also found in food processing, agriculture, and water industries.

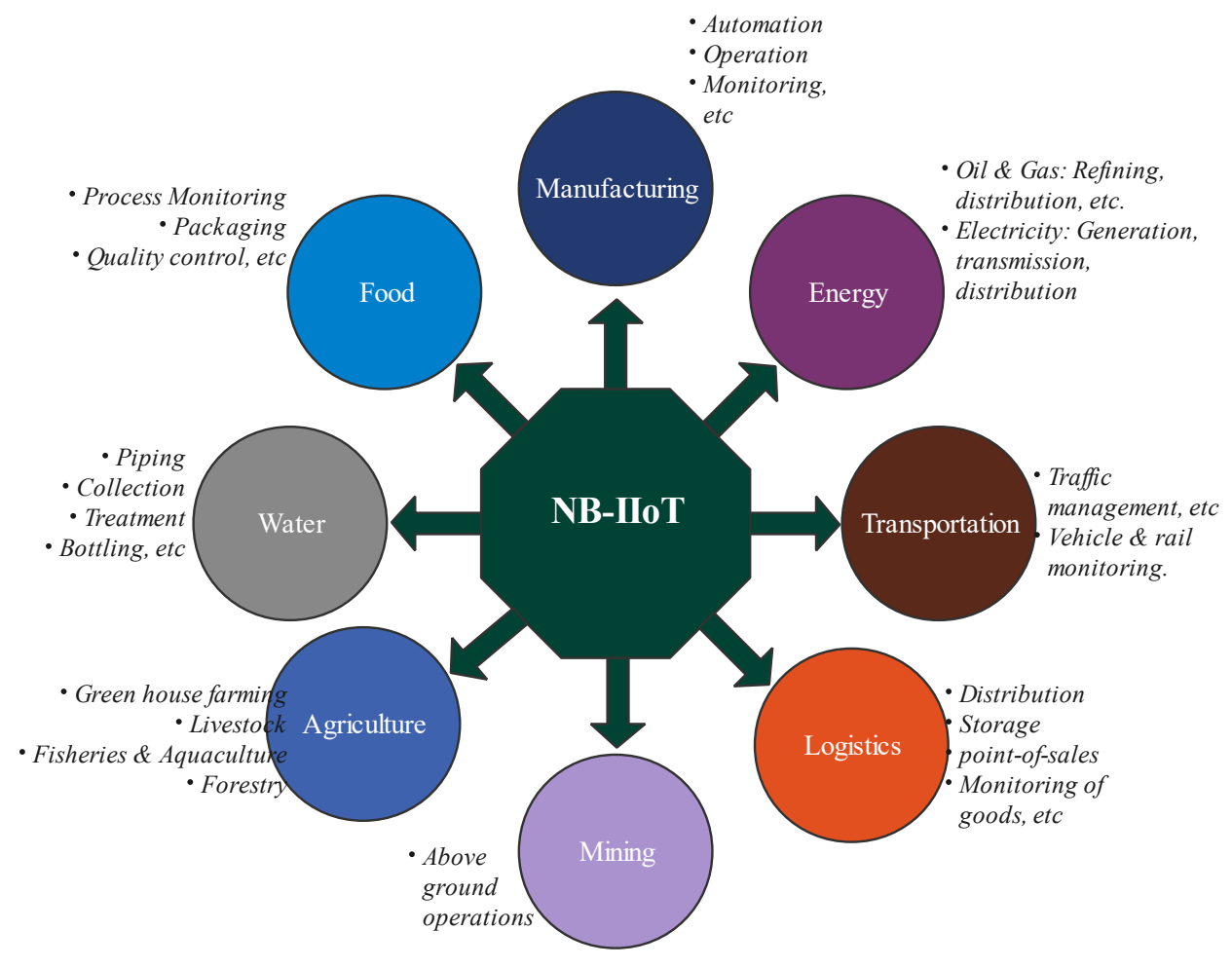

Figure 13. Areas of NB-IoT applications in industries.

\section{State-of-the-Art}

With the emphasis and success of private mobile networks in industrial settings, the NB-IoT has found its application in the industrial world-Narrow-Band Industrial Internet of Things (NB-IIoT). Its application relies on the existing infrastructure of the legacy LTE. However, the ad hoc WSN forms the bedrock of the IoT. This is because the introduction of a unifying infrastructure that simply allows the identification, addressing and communications of sensors and objects has enhanced the massive connectivity of these devices. This section is organized into two main sections. The first section is about the developmental trends and latest technological features of WSN, IoT, and NB-IoT. The second section presents the application case studies of WSN, IoT, and NB-IoT in industrial environments, respectively.

\subsection{Developmental Trends and Technical Features}

\subsubsection{The Wireless Sensor Networks}

The observation or monitoring of physical quantities has become necessary over the years. This is not only to provide useful information about the observed entity but also to enable quality decisions. These physical quantities are usually monitored by sensors or nodes. WSN gathers data from the point of observation through a storage unit to the point where decisions are made [36]. The types, applications, and topology of WSN have 
been described in [35]. The authors in [37] described how WSN can provide the functional requirements in monitoring the environment, which also brings about convenience and efficient methods as compared to the use of human capital.

\subsubsection{Internet of Things (IoT)}

A commonly acceptable consideration of IoT is where regular objects (physical and virtual, which can be identified either through their physical or virtual attributes) are connected to a scalable and unifying network infrastructure- the Internet. In other words, an infrastructure that can use some sets of regulatory communication protocols for selfconfiguration to provide a reliable and easy connection for these objects [27]. While the term "Internet of Things" was coined by Kevin Ashton in the year 1999 [28], the basic concept behind it was the combination and application of various technologies such as sensors, Radio Frequency Identification (RFID), and actuators, etc., to bring about the interaction among objects that applies these techniques in a standardized form [29]. These objects are called smart objects because of their ability to interact with the environment and other objects as well.

A survey of some factors and technologies that drives IoT was discussed in [30,31], factors such as communication links (e.g., ZigBee, Bluetooth), identification of objects through electronic product code (EPC) or ubiquitous codes (ucode), smart sensing and computational processes, among other things. The ubiquity of IoT is leading to a paradigm of its kind such as the Internet of People (IoP), Internet of Services (IoS), Internet of Content $(\mathrm{IoC})$, and Internet of Agents (IoA) [32]. Based on the relation of this paradigm with IoT, the authors analyzed these trends and the challenges ahead. The authors in $[33,34]$ reviewed the architecture, networks, and smart objects that form the building blocks of IoT.

\subsubsection{Industrial Internet of Things (IIoT)}

The dynamic application of IoT to every aspect of human activities has led to its extension in the industrial sector. This extension can be viewed as the development of IoT for the specific application in industries [22,23]. These IoT are expected to meet the unique needs of these industries. The IIoT is considered to be a subcategory of IoT that focuses on industrial communication technologies, M2M applications, etc., as explained in [21]. As a subset, the paper presented the similarities between IoT and IIoT. More so, Reference [20] sees IIoT as a technology that is used to achieve the goals of industries through the deployment of some IoT technologies in a specific way that involves the use of intelligent objects on cyber-physical platforms.

The peculiarity of industrial needs for the application of IoT, among other things, includes quality of service (QoS) [24], and the authors proposed a two-layered architectural distribution of a hybrid IIoT for QoS. One of the aims of IIoT is to provide real-time information to decision-makers about the state of the industry. To achieve this, Reference [25] developed a wireless gateway for the application of IIoT. A framework for the use of Machine Learning (ML) in the processing of generated data from IIoT has been studied in [26].

\subsubsection{Narrow-Band IoT, IIoT (NB-IoT, NB-IIoT)}

Unlike WSN, IWSN, IoT, and IIoT, the Narrow Band (NB) NB-IoT and NB-IIoT are devices that operate in licensed frequency bands. Following the Third Generation Partnership Project (3GPP), the NB-IoT has a history of standardization from 2005 when version R8 was released to 2016 with the release of version R14 [1]. Among the needs that necessitated the design of NB-IoT is the support for large numbers of IoT devices, extended coverage range due to high spectral density, low power consumption rate, low latency and reliability $[1,8]$. The coverage range of NB-IoT has been compared with other LPWAN technologies and found to reach $1 \mathrm{~km}$ in urban and up to $10 \mathrm{~km}$ in rural settlements with a low interference rate $[6,7]$. 
NB-IoT has provided requirements to support a large number of devices with a low data rate demanded by industrial operations [5]. The reliability and QoS of NB-IoT is one of the requirements and needs of the industries [9]. NB-IoT also provides ease of deployment to industries, especially in Europe where the network is more established [6].

\subsection{Application Case Studies in Industrial Environments}

\subsubsection{Industrial Applications of Wireless Sensor Networks (IWSN)}

The application of WSN in the oil and gas industries was presented in [38,39]. The authors discussed the implementation of WSN in the three major sectors of the oil and gas industries. This includes the challenges and requirements for the deployment of WSN. While WSNs are still faced with real-time reliability issues and security, Reference [40] designed and implemented a low-powered ZigBee technology used to monitor and provide a predictive maintenance routine in the oil and gas industry. An example of Industrial Wireless Sensor Network (IWSN) Automation was described in [41]. The performance metrics required for IWSN have necessitated its development as compared to the already existing technologies such as cellular communications, $\mathrm{Wi}-\mathrm{Fi}$, etc. These metrics include Low power consumption, the distance between communication points, security, service reliability, data transmission rate, and latency.

\subsubsection{Applications of Internet of Things (IoT)}

Reference [83] Surveyed the applications of IoT and mobile phone computing and categorized them based on areas of application. The authors' survey indicates a wide range of applications of IoT in the current state of technology. The applications of IoT also extend to the underground such as mining of mineral resources. In [84], the paper introduced an underground emergency hedging subsystem that aids the use of IoT technology to improve its underground application. In the agricultural field, IoT has found application in arable farming as well, which is detailed in [85]. The paper analytically reviewed the current state of IoT application in arable farming as well as the potential. The authors in [86] explained how the applications of IoT are making things smart, such as smart infrastructure, smart homes, smart traffics systems, smart healthcare, etc. The application of IoT can also be found in an electric power system where the stability of the grid can be improved using an interline power flow controller (IPFC) [87]. The authors explained how this was achieved logically. With the recent emergence and deployment of 5G technology around the world, Reference [88] provides an overview of how IoT could be integrated into the $5 \mathrm{G}$ wireless system.

The opportunities provided by IIoT through remote management are one of the main advantages the industries (manufacturers, health services, agriculture, etc.) are leveraging to improve efficiency and productivity [21]. IIoT finds its application in smart manufacturing, for effective wireless communication among IIoT devices and gateways, for example, Reference [89] proposed a Hierarchical Trustful Resource Assignment (HTRA) and Trust Computing Algorithm (TCA) to achieve this, while Reference [90] added a suite consisting of macro-services-based. To provide an effective predictive maintenance culture for Industrial types of machinery, IIoT has been deployed as a solution through the NGS-PlantOne System as detailed in [91]. The presentation of a concept for the application of IIoT in shipyards has been provided in [92]. The connection between physical devices and communication between information systems was studied.

\subsubsection{NB-IoT Industrial Application (NB-IIoT)}

NB-IoT has been used in Power Wireless Private Networks (PWPN) in power industries [11] to achieve deep and large coverage. The analysis made by the authors also shows that NB-IoT is suitable for the latency tolerance services required in PWPN. To improve the efficiency of next-generation aircraft industries, the low power consumption feature of NB-IoT has enabled its usage in aircraft industries as authored by [93]. The integration of NB-IoT into LTE functionalities has enabled this technology to co-exist with the LTE 
infrastructure, and its application in smart cities is encouraging [19]. More applications of NB-IoT are found in e-Health [94] and utility industries such as smart metering and tracking [95]. The suitability of NB-IoT deployment in smart energy distribution networks has been tested and optimized in [96].

\subsection{Challenges in Industrial Environments}

\subsubsection{WSN in Industrial Settings}

The industrial environment has always been harsh to wireless channels. The effect of this phenomenon is visible in the erroneous packet transfer in IWSN. To solve this effect, Reference [97] developed a novel Mixed Integer Programming (MIP) Framework that improves the quality of packet transfer for more battery sustainability in IWSN. The influence on the communication channel of WSN depends on the environment in which they are deployed. This applies to industrial environments as well, and the extent of influence on the channel also depends on the type of industrial setup. In [98], some challenges, such as security, routing of data, etc., were studied in addition to application types of WSN. Reference [99] discussed most of the factors that should be considered when planning WSN deployment for targeted usage. These factors include the communication range of nodes, sensing range, deployment strategy, etc. Reference [45] primarily focused on the challenge of coverage in WSNs. Among the three types of coverage (barrier, point, and area) presented, the authors discussed in detail the coverage area type. The details of which are on typical research work, classified area coverage based on the type of node and deployment, coverage detection, sensing, and communication range.

\subsubsection{IoT and Its Challenges}

The transformation of IoT has made its application in various fields seamless to some extent. While IoT has made fair progress, this was not possible without some existing and emerging challenges. Some IoT challenges are highlighted in [100] as it affects energy sustainability. The challenges facing IoT range from standardization, scalability, architecture to security, which were highlighted in [68]. Security challenges were the major aspect that Reference [101] focused on. In the paper, based on IoT layers, the authors studied the security issues facing it: requirements, limitations, as well as the way forward. Developing an application for IoT poses challenges for its smooth usage, Reference [102] presented an IoT application development framework (IADev) to mitigate this challenge. The paper described how IADev improves the framework for IoT application improvement.

\subsubsection{IIoT Challenges}

While the opportunities presented by IIoT cannot be overemphasized, IIoT is still faced with challenges, some of which have received attention in recent years. Reference [21] highlight these challenges. Reference [56] discussed the challenges faced in the application of IoT in logistics industries. These challenges are based on common IoT structural design, which includes network topology, the latency of packets, throughput, power sustainability, the security of data, and scalability of the technology. To solve the latency issue as one of the challenges facing IIoT, Reference[103] proposed a low-latency distributed scheduling function (LDSF). Reliability and latency are important requirements in IIoT for industrial environments. The authors in [104] studied the impact of physical, media access control, and network layers on these two requirements. One of the impacting factors on IIoT in industrial environments is electromagnetic noise. To overcome this, Reference [105] collected electromagnetic noise data from an automobile factory by measuring and characterizing the data for IIoT systems.

\subsubsection{NB-IoT in Industries}

Areas that are difficult to cover with NB-IoT devices experience bad channel conditions and to mitigate this, rigorous re-transmission or repetition is required. This process requires more energy, which, in turn, reduces the battery life of the devices. More so, there 
is a restriction regarding large numbers of devices being deployed, which is a result of the unclear performance metrics, such as coverage range, throughput, etc., required in an NB-IoT-supported deployment (guard-band, in-band and standalone) [17]. In [106], the authors analyzed the security issues faced by NB-IoT in a layered manner, which provides substantially different security attack proposals on these layers. NB-IoT technology is applicable in most aspects of an industrial operation; however, to some extent, with wireless deployment, the expectations of factory enhancement fail, especially in the areas of latency, reliability, and resilience. Reference [107] provides problem category classification where the deployment of network technologies is achievable. Generally, intensive interference, high noise levels, and shadowing are some of the factors that make the industrial environment harsh to radio or wireless communications. To overcome these, Reference [108] presented measurements for path-loss and shadowing at 900, 1600, and $2450 \mathrm{MHz}$ to enable proper wireless channel design for industrial setups. The future applications of NB-IoT in health industries as well as the challenges faced are discussed in [109]. To harvest the full potential of next-generation cloud computing, Reference [110] studied the possible challenges that need to be overcome, infusing cloud computing infrastructure, connecting people and devices through IoT.

\section{Future Research Prospects}

Industrial processes undergo several different stages for the final production of goods and services. The continuous efforts in the development and integrations of NB-IoT applications in industries are welcomed in the broader world of IoT. More so, since the involvement of NB-IoT in industrial activities requires a huge number of its deployment for process monitoring and data gathering, many of the solutions provided by vendors are yet to fully satisfy the requirements of the industrial world.

Among the key requirements of industrial settings are: NB-IoT as a Self-Organizing Network, leveraging on edge computing to improve scalability in the deployment of an NB-IoT network, assurance in the security of industrial data generated, and having a suitable NB-IoT model that meets its propagation challenges.

\subsection{Self-Organizing Network (SON) and NB-IoT Management}

The use of NB-IoT in industries has made activities easy, safe, effective, and more productive. However, as the number grows, the management of these devices becomes cumbersome and complex. To achieve a continuous and smooth production level, more physical efforts are required to maintain these devices. While some attempt has been made to establish network management systems (NMS), this form of management still requires physical configuration, registration, and maintenance of devices whenever they breakdown or are introduced into the network system. The management of NB-IoT is not limited to device management where provision for automatic control, including firmware upgrade, activation and deactivation, and monitoring capabilities, is required, but also network management functionalities are required to maintain network topology efficiency, synchronization of devices, and traffic congestion control. Data management can not be left out as this involves the ability to aggregate raw data for data analytics and data recovery [111].

Since NB-IoT is a superimposition on LTE and its applications are constantly increasing in number, all the main drivers of SON that apply to LTE are also applicable to NBIoT. These drivers include complexity and a large number of NB-IoT structured network parameters, an increase in the number of base stations (eNB) to which NB-IoT devices are attached, and a requirement of regular human intervention for configurations and management [112]. In Figure 14, the main functionalities of SON are explained as related to NB-IoT applications. The first process involves self-configuration, a process where necessary and basic configurations for system operation are automatically performed for newly deployed modules. This process starts with a stage called self-learning where parameters, such as the NB-IoT network provider, network registration status, location 
information, home subscriber servers, signal connection status viz idle or connected, and update on NB-IoT PSM, are all learned. This is followed by the configurations of the operational parameters, which include: eDRx, PSM, cell selection, coverage extension, Physical Resource Block (PRB), and firmware upgrade. The second process is the selfoptimization process, which involves monitoring of events and performance optimization. NB-IoT uses the Service Capabilities Exposure Function (SCEF) deployment plan to monitor events, and these events include: roaming, loss of connection, traffic types viz critical, remote, and non-critical traffics, and signal quality. The performance optimization stage optimizes data transportation in the control plane Cellular-IoT (CIoT) Evolved Packet System (EPS), rate control for quality assurance, roaming for hand-off, multi-frequency band, and deployment mode for bandwidth. The third process of SON is self-healing, where paging and power class are used for outage and power maintenance. NB-IoT employs SCEF plans for User Equipment (UE) reachability, location, and change in location of the UE, communication failure, availability after DDN failure, and change of IMSI-IMEI (SV) associations [113].

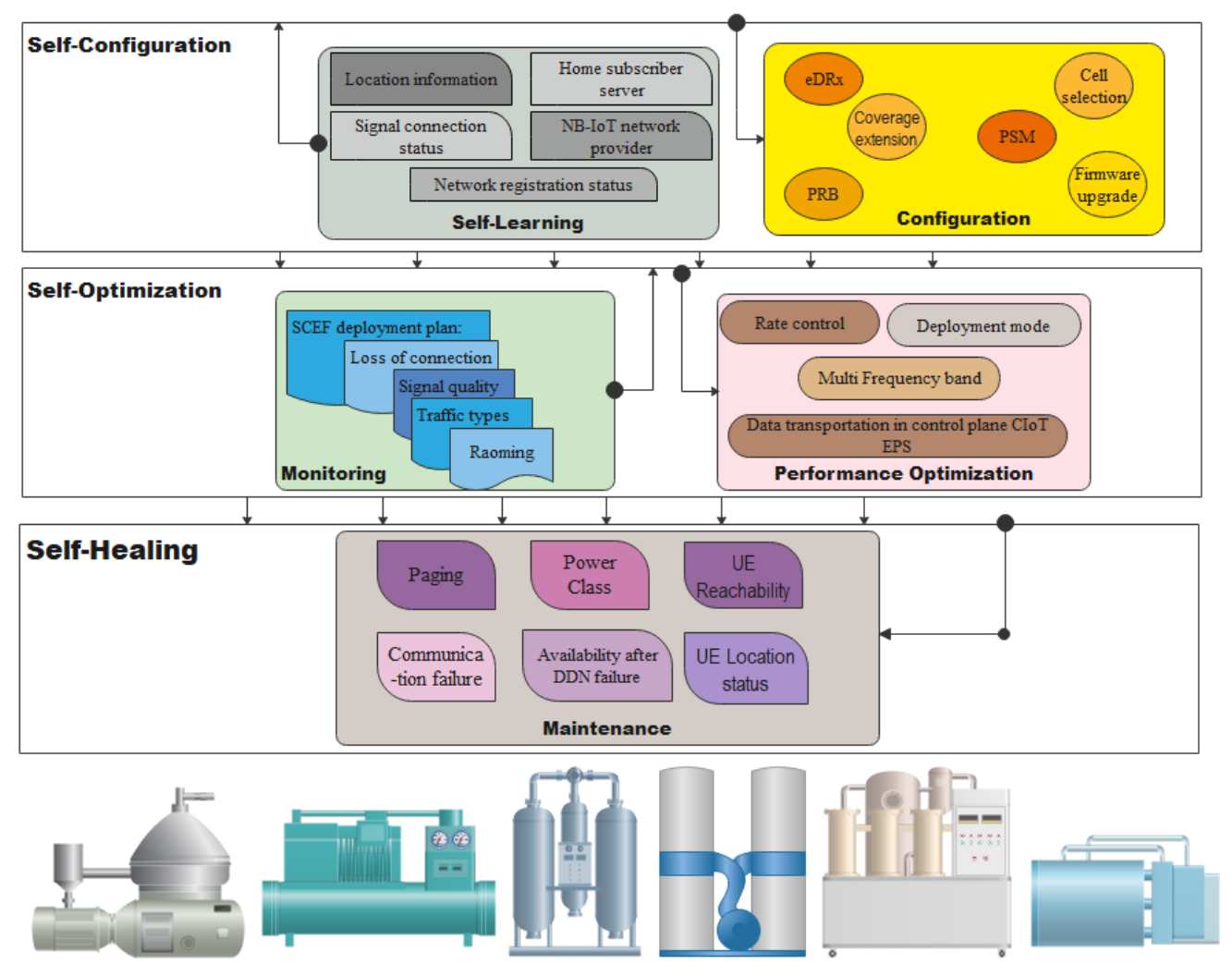

NB-Industrial Internet of Things and Data Generators

Figure 14. Application of SON in NB-IoT networks.

\subsection{Edge Computing and Scalability in NB-IoT Network}

Scalability in an NB-IoT network requires the introduction of new devices into the network without any reduction in the network performance and minimum human intervention. To achieve any level of scalability in the network, some challenges have to be resolved. The interoperability among devices deployed, memory space needed for data storage, the processing power of the system, and bandwidth for smooth and effective data transmission are all parts of the key challenges that need to be resolved for efficient scalability [114].

In order to mitigate some of these challenges, the edge computing technology can be deployed to provide a more robust and cost effective computing power in terms of memory, speed, and enhanced scalability to the NB-IoT network. Edge computing, however, is an 
intermediary between the NB-IoT network and cloud computing. Therefore, this proposed system will require a stable and reliable cloud computing platform that supports NB-IoT for industrial cases [115]. Resolving these challenges will go a long way to build the confidence of industrial managers in the implementation of NB-IoT networks.

\subsection{Security of Data in Industrial NB-IoT}

Protecting data from unauthorized access and corruption throughout its life cycle is a huge concern to every industrial management team. Therefore, securing NB-IoT data generation in an industrial environment incorporates a complex set of industrial processes and practices that needs to accommodate both reliability and safety requirements [50]. These requirements are not limited to but include the following: confidentiality and integrity of data and users. This can be achieved through a protection policy that allows secured, encrypted, version control, error detection, backup and recovery procedure mechanisms, password management, two-factor authentication, biometric verification, and access control for users. This is because confidentiality and integrity in data allows accurate, complete, and unmodified data to be accessed by only approved users, a requirement that ensures the continued survival of industrial practice. Service availability is a requirement that ensures easy access to NB-IoT data whenever they are needed. To meet this requirement, NB-IoT data must incorporate a mechanism that supports off-sight back-ups or mirroring, a quick and safe data recovery system, and a redundancy mechanism to avoid complete network failover. However, to avoid data breaches and failures, a constant and proper monitoring policy needs to be put in place by industrial management. Data freshness is another requirement paramount to NB-IoT data security, owing to the limitation in the memory capacity of these modules and the need to have up-to-date data for decision making using the generated data. To overcome this challenge, a secured Cloud Internet of Things framework can be deployed to provide this memory space requirement.

\subsection{Suitable NB-IoT Industrial Propagation Model}

Since the industrial wireless environments are characterized by high reflectiveness, which causes multi-path effect and electromagnetic resonance, this phenomenon creates significant harshness on wireless communication in indoor industrial environments [116]. While every industry is unique in its setup, many works of literature have presented propagation models that attempt to adequately describe the characteristics of wireless propagation in industries [3,107]. Understanding the wireless characteristics of an indoor industrial environment in terms of its stringent quality of service (QoS) requirements is necessary and makes it easy to plan and design wireless systems suitable for industrial applications. A comprehensive comparative analysis of channel models among lowpowered and high-powered IoT (including NB-IoT) for industrial applications, and a generic propagation path-loss (PL) for $900 \mathrm{MHz}$ application for the indoor industrial environment was presented as shown in Equation (1) [4].

$$
P L=61.65+24.9 \log _{10}\left(d_{3 D} / 15\right), \sigma=7.35
$$

Therefore, to the best of our knowledge in this survey, the wireless propagation of NB-IoT in an indoor industrial environment still requires further research that investigates the latency, reliability, and coverage characteristics of its propagation. Further research in this respect is already ongoing, and subsequent addressing of these challenges will give the industry confidence in the wireless connectivity of their devices and processes.

The success of NB-IoT and the subsequent decentralization of industrial processes will allow rearrangement of the hierarchy of industrial flows, information and processes leading to a more productive environment. This is where we believe state-of-the-art distributed ledger technologies in harmony with edge computing will allow for rich analytical information that could unlock industrial processes that were never even considered before [10]. For instance, in addition to the further directions discussed above, blockchain-based offerings looking to disintermediate the business of outsourcing storage and computing will break 
the monopoly of expensive, wasteful and environmentally harmful dominance of corporate data-centers, bringing full control of processes to local industries. Research efforts in these areas that decentralize the data, analyze it using machine learning techniques and then use edge computing for real-time analytics will unlock several industrial applications.

\section{Conclusions}

In this work, we presented a review of the Narrow-Band Internet of Things (NBIoT) and its application in industries (NB-IIoT). The reviews of Wireless Sensor Networks (WSN), as well as IoT, were made. These were presented in a manner that shows the relationships between these technologies. The review, among other things, covers the technological features, applications, and challenges. A novel presentation of the transmission tone mechanism of NB-IoT was made. Comparisons were made between NB-IoT and other Low Power Wide Area Networks (LPWAN). The paradigm presented by NB-IoT allows a brief and unique pictorial overview of its standardization process as provided by 3GPP. While NB-IoT is an integration into the legacy LTE and increases its application in industries, this work presents the wireless communication challenges that the technology faces in the industrial environment. As a future research direction, we present the need for future research into wireless communication of NB-IoT in industrial environments. These include the need for NB-IoT networks to be self-organizing in a manner that meets the industrial requirements. Furthermore, we also discussed the application of edge computing to improve the scalability of the network management, securing the data to maintain confidentiality, integrity, and availability, and finally, providing a suitable propagation model that describes the industrial wireless system.

Author Contributions: Conceptualization, M.D.; methodology, M.D.; software, M.D. and S.A.; formal analysis, S.A. and S.H.; resources, S.A., S.H. and Q.H.A.; writing-original draft preparation, M.D.; writing—review and editing, M.D., S.A., S.H. and Q.H.A; visualization, M.D.; supervision, S.H. and M.A.I.; project administration, S.H. and M.A.I.; funding acquisition, Q.H.A. All authors have read and agreed to the published version of the manuscript.

Funding: This research was funded by PTDF 1671/19 and the APC was funded by EPSRC EP/R511705/1.

Institutional Review Board Statement: Not applicable.

Informed Consent Statement: Not applicable.

Data Availability Statement: Not applicable.

Acknowledgments: This work is supported by the Petroleum Technology Development Fund (PTDF) of the Federal Republic of Nigeria with Grant No.: 1671/19 and also in part by EPSRC EP/R511705/1.

Conflicts of Interest: The authors declare no conflict of interest.

\section{References}

1. Chen, M.; Miao, Y.; Hao, Y.; Hwang, K. Narrow Band Internet of Things. IEEE Access 2017, 5, 20557-20577. [CrossRef]

2. Number of Internet of Things (IoT) Connected Devices Worldwide in 2018, 2025 and 2030 (In Billions). 2019. Available online: https:/ / www.statista.com/statistics/802690/worldwide-connected-devices-by-access-technology/ (accessed on 10 August 2020).

3. Tanghe, E.; Joseph, W.; Verloock, L.; Martens, L.; Capoen, H.; Herwegen, K.V.; Vantomme, W. The industrial indoor channel: Large-scale and temporal fading at 900, 2400, and $5200 \mathrm{MHz}$. IEEE Trans. Wirel. Commun. 2008, 7, 2740-2751. [CrossRef]

4. Wang, W.; Capitaneanu, S.L.; Marinca, D.; Lohan, E.S. Comparative Analysis of Channel Models for Industrial IoT Wireless Communication. IEEE Access 2019, 7, 91627-91640. [CrossRef]

5. Al-Rubaye, S.; Rodriguez, J.; Fragonara, L.Z.; Theron, P.; Tsourdos, A. Unleash Narrowband Technologies for Industrial Internet of Things Services. IEEE Netw. 2019, 33, 16-22. [CrossRef]

6. Mekki, K.; Bajic, E.; Chaxel, F.; Meyer, F. A comparative study of LPWAN technologies for large-scale IoT deployment. ICT Express 2019, 5, 1-7. [CrossRef]

7. Sinha, R.S.; Wei, Y.; Hwang, S.H. A survey on LPWA technology: LoRa and NB-IoT. ICT Express 2017, 3, 14-21. [CrossRef]

8. Adhikary, A.; Lin, X.; Wang, Y.E. Performance Evaluation of NB-IoT Coverage. In Proceedings of the 2016 IEEE 84th Vehicular Technology Conference (VTC-Fall), Montreal, QC, Canada, 18-21 September 2016; pp. 1-5. 
9. Zayas, A.D.; Merino, P. The 3GPP NB-IoT system architecture for the Internet of Things. In Proceedings of the 2017 IEEE International Conference on Communications Workshops (ICC Workshops), Paris, France, 21-25 May 2017. [CrossRef]

10. Imran, M.A.; Hussain, S.; Abbasi, Q.H., Narrowband Internet of Things (NB-IoT) for Industrial Automation. In Wireless Automation as an Enabler for the Next Industrial Revolution; Wiley: Hoboken, NJ, USA, 2020; pp. 65-87. [CrossRef]

11. Bao, L.; Wei, L.; Jiang, C.; Miao, W.; Guo, B.; Li, W.; Cheng, X.; Liu, R.; Zou, J. Coverage Analysis on NB-IoT and LoRa in Power Wireless Private Network. Procedia Comput. Sci. 2018, 131, 1032-1038. [CrossRef]

12. Azari, A.; Stefanović, D.; Popovski, P.; Cavdar, C. On the Latency-Energy Performance of NB-IoT Systems in Providing Wide-Area IoT Connectivity. IEEE Trans. Green Commun. Netw. 2020, 4, 57-68. [CrossRef]

13. Sultania, A.K.; Zand, P.; Blondia, C.; Famaey, J. Energy Modeling and Evaluation of NB-IoT with PSM and eDRX. In Proceedings of the 2018 IEEE Globecom Workshops (GC Wkshps), Abu Dhabi, United Arab Emirates, 9-13 December 2018; pp. 1-7.

14. Popli, S.; Jha, R.K.; Jain, S. A Survey on Energy Efficient Narrowband Internet of Things (NBIoT): Architecture, Application and Challenges. IEEE Access 2019, 7, 16739-16776. [CrossRef]

15. Chen, J.; Hu, K.; Wang, Q.; Sun, Y.; Shi, Z.; He, S. Narrowband Internet of Things: Implementations and Applications. IEEE Internet Things J. 2017, 4, 2309-2314. [CrossRef]

16. Lauridsen, M.; Nguyen, H.; Vejlgaard, B.; Kovacs, I.Z.; Mogensen, P.; Sorensen, M. Coverage Comparison of GPRS, NB-IoT, LoRa, and SigFox in a $7800 \mathrm{~km}^{2}$ Area. In Proceedings of the 2017 IEEE 85th Vehicular Technology Conference (VTC Spring), Sydney, NSW, Australia, 4-7 June 2017, pp. 1-5. [CrossRef]

17. Mwakwata, C.B.; Malik, H.; Alam, M.M.; Moullec, Y.L.; Parand, S.; Mumtaz, S. Narrowband Internet of Things (NB-IoT): From Physical (PHY) and Media Access Control (MAC) Layers Perspectives. Sensors 2019, 19, 2613. [CrossRef]

18. Yu, C.; Yu, L.; Wu, Y.; He, Y.; Lu, Q. Uplink scheduling and link adaptation for narrowband Internet of Things systems. IEEE Access 2017, 5, 1724-1734. [CrossRef]

19. Soussi, M.E.; Zand, P.; Pasveer, F.; Dolmans, G. Evaluating the Performance of eMTC and NB-IoT for Smart City Applications. In Proceedings of the 2018 IEEE International Conference on Communications (ICC), Kansas City, MO, USA, 20-24 May 2018; pp. 1-7. [CrossRef]

20. Boyes, H.; Hallaq, B.; Cunningham, J.; Watson, T. The industrial internet of things (IIoT): An analysis framework. Comput. Ind. 2018, 101, 1-12. [CrossRef]

21. Sisinni, E.; Saifullah, A.; Han, S.; Jennehag, U.; Gidlund, M. Industrial internet of things: Challenges, opportunities, and directions. IEEE Trans. Ind. Inform. 2018, 14, 4724-4734. [CrossRef]

22. Reddy, B.R.; Sujith, A. A comprehensive literature review on data analytics in IIoT (Industrial Internet of Things). HELIX 2018, 8, 2757-2764.

23. Liao, Y.; Loures, E.d.F.R.; Deschamps, F. Industrial Internet of Things: A systematic literature review and insights. IEEE Internet Things J. 2018, 5, 4515-4525. [CrossRef]

24. Huang, X. Quality of service optimization in wireless transmission of industrial Internet of Things for intelligent manufacturing. Int. J. Adv. Manuf. Technol. 2019, 107, 1007-1016. [CrossRef]

25. Ferreira, I.V.; Bigheti, J.A.; Godoy, E.P. Development of a wireless gateway for industrial internet of things applications. IEEE Lat. Am. Trans. 2019, 17, 1637-1644. [CrossRef]

26. Khan, A.I.; Al-Badi, A. Open Source Machine Learning Frameworks for Industrial Internet of Things. Procedia Comput. Sci. 2020, 170, 571-577. [CrossRef]

27. Van Kranenburg, R. A Critique of Ambient Technology and the All-Seeing Network of RFID; The Netherlands Institute of Network Culture: Amsterdam, The Netherlands, 2008.

28. Routh, K.; Pal, T. A survey on technological, business and societal aspects of Internet of Things by Q3, 2017. In Proceedings of the 2018 3rd International Conference On Internet of Things: Smart Innovation and Usages (IoT-SIU), Bhimtal, India, 23-24 February 2018; pp. 1-4.

29. Kaur, K. A Survey on internet of things-Architecture, applications, and future trends. In Proceedings of the 2018 First International Conference on Secure Cyber Computing and Communication (ICSCCC), Jalandhar, India, 15-17 December 2018; pp. 581-583.

30. Paul, P.V.; Saraswathi, R. The Internet of Things-A comprehensive survey. In Proceedings of the 2017 International Conference on Computation of Power, Energy Information and Commuincation (ICCPEIC), Melmaruvathur, India, 22-23 March 2017; pp. 421-426.

31. Solapure, S.S.; Kenchannavar, H. Internet of Things: A survey related to various recent architectures and platforms available. In Proceedings of the 2016 International Conference on Advances in Computing, Communications and Informatics (ICACCI), Jaipur, India, 21-24 September 2016; pp. 2296-2301.

32. Pico-Valencia, P.; Holgado-Terriza, J.A.; Quiñónez-Ku, X. A Brief Survey of the Main Internet-Based Approaches. An Outlook from the Internet of Things Perspective. In Proceedings of the 2020 3rd International Conference on Information and Computer Technologies (ICICT), San Jose, CA, USA, 9-12 March 2020; pp. 536-542.

33. Sabry, S.S.; Qarabash, N.A.; Obaid, H.S. The Road to the Internet of Things: A Survey. In Proceedings of the 2019 9th Annual Information Technology, Electromechanical Engineering and Microelectronics Conference (IEMECON), Jaipur, India, 13-15 March 2019; pp. 290-296. 
34. Varshini, R.; Karthikeyan, A. Internet of Things-Evolution, Architecture and Real Time Application-Survey. In Proceedings of the 2019 International Conference on Vision Towards Emerging Trends in Communication and Networking (ViTECoN), Vellore, India, 30-31 March 2019; pp. 1-4.

35. Singh, M.K.; Amin, S.I.; Imam, S.A.; Sachan, V.K.; Choudhary, A. A Survey of Wireless Sensor Network and its types. In Proceedings of the 2018 International Conference on Advances in Computing, Communication Control and Networking (ICACCCN), Greater Noida, India, 12-13 October 2018; pp. 326-330. [CrossRef]

36. Deepika, G.; Rajapirian, P. Wireless sensor network in precision agriculture: A survey. In Proceedings of the 2016 International Conference on Emerging Trends in Engineering, Technology and Science (ICETETS), Solan, India, 20-22 December 2016; pp. 1-4.

37. Othman, M.F.; Shazali, K. Wireless sensor network applications: A study in environment monitoring system. Procedia Eng. 2012, 41, 1204-1210. [CrossRef]

38. Aalsalem, M.Y.; Khan, W.Z.; Gharibi, W.; Khan, M.K.; Arshad, Q. Wireless Sensor Networks in oil and gas industry: Recent advances, taxonomy, requirements, and open challenges. J. Netw. Comput. Appl. 2018, 113, 87-97. [CrossRef]

39. Aalsalem, M.Y.; Khan, W.Z.; Gharibi, W.; Armi, N. An intelligent oil and gas well monitoring system based on Internet of Things. In Proceedings of the 2017 International Conference on Radar, Antenna, Microwave, Electronics, and Telecommunications (ICRAMET), Jakarta, Indonesia, 23-24 October 2017; pp. 124-127. [CrossRef]

40. Khakpour, K.; Shenassa, M. Industrial control using wireless sensor networks. In Proceedings of the 2008 3rd International Conference on Information and Communication Technologies: From Theory to Applications, Damascus, Syria, 7-11 April 2008; pp. 1-5.

41. Zheng, L. Industrial wireless sensor networks and standardizations: The trend of wireless sensor networks for process autometion. In Proceedings of the SICE Annual Conference 2010, Taipei, Taiwan, 18-21 August 2010; pp. 1187-1190.

42. Akyildiz, I.F.; Su, W.; Sankarasubramaniam, Y.; Cayirci, E. A survey on sensor networks. IEEE Commun. Mag. 2002, 40, 102-114. [CrossRef]

43. Sachan, V.K.; Imam, S.A.; Beg, M. Energy-efficient communication methods in wireless sensor networks: A critical review. Int. J. Comput. Appl. 2012, 39, 35-48.

44. Intanagonwiwat, C.; Govindan, R.; Estrin, D. Directed diffusion: A scalable and robust communication paradigm for sensor networks. In Proceedings of the 6th Annual International Conference on Mobile Computing and Networking, Boston, MA, USA, 1 August 2000; pp. 56-67.

45. Singh, A.; Sharma, T. A survey on area coverage in wireless sensor networks. In Proceedings of the 2014 International Conference on Control, Instrumentation, Communication and Computational Technologies (ICCICCT), Kanyakumari, India, 10-11 July 2014; pp. 829-836.

46. Swetha, R.; Amarnath, S.; Sofia, A. Wireless Sensor Network: A Survey. IJARCCE Int. J. Adv. Res. Comput. Commun. Eng. 2018, 7, 11. [CrossRef]

47. Gungor, V.C.; Lu, B.; Hancke, G.P. Opportunities and challenges of wireless sensor networks in smart grid. IEEE Trans. Ind. Electron. 2010, 57, 3557-3564. [CrossRef]

48. Swati, A.J.; Priyanka, R. Wireless sensor network (WSN): Architectural design issues and challenges. Int. J. Comput. Sci. Eng. 2010, 2, 3089-3094.

49. Schoder, D. Introduction to the Internet of Things. In Introduction to the Internet of Things. Internet of Things A to Z: Technologies and Applications; Wiley: Hoboken, NJ, USA, 2018; pp. 3-50.

50. Mumtaz, S.; Alsohaily, A.; Pang, Z.; Rayes, A.; Tsang, K.F.; Rodriguez, J. Massive Internet of Things for industrial applications: Addressing wireless IIoT connectivity challenges and ecosystem fragmentation. IEEE Ind. Electron. Mag. 2017, 11, 28-33. [CrossRef]

51. Chaouchi, H. The Internet of Things: Connecting Objects to the Web; John Wiley \& Sons: Hoboken, NJ, USA, 2013.

52. Khan, W.; Rehman, M.; Zangoti, H.; Afzal, M.; Armi, N.; Salah, K. Industrial internet of things: Recent advances, enabling technologies and open challenges. Comput. Electr. Eng. 2020, 81, 106522. [CrossRef]

53. Jamali, J.; Bahrami, B.; Heidari, A.; Allahverdizadeh, P.; Norouzi, F. Towards the Internet of Things; Springer International Publishing AG: Cham, Switzerland, 2020.

54. Ngu, A.H.; Gutierrez, M.; Metsis, V.; Nepal, S.; Sheng, Q.Z. IoT middleware: A survey on issues and enabling technologies. IEEE Internet Things J. 2016, 4, 1-20. [CrossRef]

55. Ning, H.; Wang, Z. Future internet of things architecture: Like mankind neural system or social organization framework? IEEE Commun. Lett. 2011, 15, 461-463. [CrossRef]

56. Flügel, C.; Gehrmann, V. Scientific workshop 4: Intelligent objects for the internet of things: Internet of things-application of sensor networks in logistics. In Constructing Ambient Intelligence (AmI 2008). Communications in Computer and Information Science; Gerhäuser, H., Hupp, J., Efstratiou, C., Heppner, J., Eds.; Springer: Berlin/Heidelberg, Germany, 2008; Volume 32. [CrossRef]

57. Finnegan, J.; Brown, S. A comparative survey of LPWA networking. arXiv 2018, arXiv:1802.04222.

58. Akpakwu, G.A.; Silva, B.J.; Hancke, G.P.; Abu-Mahfouz, A.M. A survey on 5G networks for the Internet of Things: Communication technologies and challenges. IEEE Access 2017, 6, 3619-3647. [CrossRef]

59. Raza, M.; Aslam, N.; Le-Minh, H.; Hussain, S.; Cao, Y.; Khan, N.M. A critical analysis of research potential, challenges, and future directives in industrial wireless sensor networks. IEEE Commun. Surv. Tutor. 2017, 20, 39-95. [CrossRef] 
60. Centenaro, M.; Vangelista, L.; Zanella, A.; Zorzi, M. Long-range communications in unlicensed bands: The rising stars in the IoT and smart city scenarios. IEEE Wirel. Commun. 2016, 23, 60-67. [CrossRef]

61. Mecheraoui, C.; Cobb, J.; Swain, I. Evaluation of a wireless in-shoe sensor based on ZigBee used for drop foot stimulation. In Proceedings of the 2012 IEEE Radio and Wireless Symposium, Santa Clara, CA, USA, 15-18 January 2012; pp. $423-426$.

62. Treurniet, J.J.; Sarkar, C.; Prasad, R.V.; De Boer, W. Energy consumption and latency in BLE devices under mutual interference: An experimental study. In Proceedings of the 2015 3rd International Conference on Future Internet of Things and Cloud, Rome, Italy, 24-26 August 2015; pp. 333-340.

63. Proskochylo, A.; Vorobyov, A.; Zriakhov, M.; Kravchuk, A.; Akulynichev, A.; Lukin, V. Overview of wireless technologies for organizing sensor networks. In Proceedings of the 2015 Second International Scientific-Practical Conference Problems of Infocommunications Science and Technology (PIC S\&T), Kharkiv, Ukraine, 13-15 October 2015; pp. 39-41.

64. Lee, J.; Su, Y.; Shen, C. A Comparative Study of Wireless Protocols: Bluetooth, UWB, ZigBee, and Wi-Fi. In Proceedings of the IECON 2007-33rd Annual Conference of the IEEE Industrial Electronics Society, Taipei, Taiwan, 5-8 November 2007 ; pp. 46-51.

65. Mulla, A.Y.; Baviskar, J.J.; Kazi, F.S.; Wagh, S.R. Implementation of ZigBee/802.15.4 in Smart Grid communication and analysis of power consumption: A case study. In Proceedings of the 2014 Annual IEEE India Conference (INDICON), Pune, India, 11-13 December 2014; pp. 1-7.

66. Xu, L.D.; He, W.; Li, S. Internet of Things in Industries: A Survey. IEEE Trans. Ind. Inform. 2014, 10, 2233-2243. [CrossRef]

67. Lin, J.; Yu, W.; Zhang, N.; Yang, X.; Zhang, H.; Zhao, W. A Survey on Internet of Things: Architecture, Enabling Technologies, Security and Privacy, and Applications. IEEE Internet Things J. 2017, 4, 1125-1142. [CrossRef]

68. Goswami, S.A.; Padhya, B.P.; Patel, K.D. Internet of Things: Applications, Challenges and Research Issues. In Proceedings of the 2019 Third International conference on I-SMAC (IoT in Social, Mobile, Analytics and Cloud) (I-SMAC), Palladam, India, 12-14 December 2019; pp. 47-50.

69. Miorandi, D.; Sicari, S.; De Pellegrini, F.; Chlamtac, I. Internet of things: Vision, applications and research challenges. Ad Hoc Netw. 2012, 10, 1497-1516. [CrossRef]

70. Lee, S.K.; Bae, M.; Kim, H. Future of IoT Networks: A Survey. Appl. Sci. 2017, 7, 1072. [CrossRef]

71. Ferrari, P.; Flammini, A.; Sisinni, E.; Rinaldi, S.; Brandão, D.; Rocha, M.S. Delay Estimation of Industrial IoT Applications Based on Messaging Protocols. IEEE Trans. Instrum. Meas. 2018, 67, 2188-2199. [CrossRef]

72. Celebi, H.B.; Pitarokoilis, A.; Skoglund, M., Wireless Communication for the Industrial IoT. In Industrial IoT : Challenges, Design Principles, Applications, and Security; Butun, I., Ed.; Springer International Publishing: Cham, Switzerland, 2020 ; pp. 57-94.

73. Voigtländer, F.; Ramadan, A.; Eichinger, J.; Lenz, C.; Pensky, D.; Knoll, A. 5G for Robotics: Ultra-Low Latency Control of Distributed Robotic Systems. In Proceedings of the 2017 International Symposium on Computer Science and Intelligent Controls (ISCSIC), Budapest, Hungary, 20-22 October 2017; pp. 69-72.

74. Sasaki, K.; Makido, S.; Nakao, A. Vehicle Control System for Cooperative Driving Coordinated Multi -Layered Edge Servers. In Proceedings of the 2018 IEEE 7th International Conference on Cloud Networking (CloudNet), Tokyo, Japan, 22-24 October 2018; pp. 1-7.

75. Gubbi, J.; Buyya, R.; Marusic, S.; Palaniswami, M. Internet of Things (IoT): A vision, architectural elements, and future directions. Future Gener. Comput. Syst. 2013, 29, 1645-1660. [CrossRef]

76. Pöhls, H.C.; Angelakis, V.; Suppan, S.; Fischer, K.; Oikonomou, G.; Tragos, E.Z.; Rodriguez, R.D.; Mouroutis, T. RERUM: Building a reliable IoT upon privacy- and security- enabled smart objects. In Proceedings of the 2014 IEEE Wireless Communications and Networking Conference Workshops (WCNCW), Istanbul, Turkey, 6-9 April 2014; pp. 122-127.

77. Peter, H.C.; Damith, C.R., Rerum: Building a reliable IoT upon privacy-and security-enabled smart objects. In Networked RFID Systems and Lightweight Cryptography; Peter, H.C.; Damith, C.R., Eds.; Springer International Publishing: Cham, Switzerland, 2007; pp. 57-94.

78. Hoymann, C.; Astely, D.; Stattin, M.; Wikstrom, G.; Cheng, J.; Hoglund, A.; Frenne, M.; Blasco, R.; Huschke, J.; Gunnarsson, F. LTE release 14 outlook. IEEE Commun. Mag. 2016, 54, 44-49. [CrossRef]

79. NarrowBand IoT Wide Range of Opportunities. 2020. Available online: https://www-file.huawei.com/-/media/corporate/ minisite/mwc2016/pdf/narrowband-iot-wide-range-of-opportunities-en.pdf?la=en (accessed on 17 June 2020).

80. Andres-Maldonado, P.; Ameigeiras, P.; Prados-Garzon, J.; Navarro-Ortiz, J.; Lopez-Soler, J.M. An Analytical Performance Evaluation Framework for NB-IoT. IEEE Internet Things J. 2019, 6, 7232-7240. [CrossRef]

81. Ballerini, M.; Polonelli, T.; Brunelli, D.; Magno, M.; Benini, L. NB-IoT vs. LoRaWAN: An Experimental Evaluation for Industrial Applications. IEEE Trans. Ind. Inform. 2020, 16, 7802-7811. [CrossRef]

82. Ballerini, M.; Polonelli, T.; Brunelli, D.; Magno, M.; Benini, L. Experimental Evaluation on NB-IoT and LoRaWAN for Industrial and IoT Applications. In Proceedings of the 2019 IEEE 17th International Conference on Industrial Informatics (INDIN), Helsinki, Finland, 22-25 July 2019; Volume 1, pp. 1729-1732.

83. Kamilaris, A.; Pitsillides, A. Mobile Phone Computing and the Internet of Things: A Survey. IEEE Internet Things J. 2016, 3, 885-898. [CrossRef]

84. Wang, J.; Guo, Y.; Jia, Y.; Zhang, Y.; Li, M. Modeling and Application of the Underground Emergency Hedging System Based on Internet of Things Technology. IEEE Access 2019, 7, 63321-63335. [CrossRef]

85. Villa-Henriksen, A.; Edwards, G.T.; Pesonen, L.A.; Green, O.; Sørensen, C.A.G. Internet of Things in arable farming: Implementation, applications, challenges and potential. Biosyst. Eng. 2020, 191, 60-84. [CrossRef] 
86. Goyal, P.; Sahoo, A.K.; Sharma, T.K.; Singh, P.K. Internet of Things: Applications, security and privacy: A survey. Mater. Today Proc. 2020, 34, 752-759. [CrossRef]

87. Radhakrishnan, G.; Gopalakrishnan, V. Applications of internet of things (IOT) to improve the stability of a grid connected power system using interline power flow controller. Microprocess. Microsyst. 2020, 76, 103038. [CrossRef]

88. Chettri, L.; Bera, R. A Comprehensive Survey on Internet of Things (IoT) Toward 5G Wireless Systems. IEEE Internet Things J. 2020, 7, 16-32. [CrossRef]

89. Xu, X.; Han, M.; Nagarajan, S.M.; Anandhan, P. Industrial Internet of Things for smart manufacturing applications using hierarchical trustful resource assignment. Comput. Commun. 2020, 160, 423-430. [CrossRef]

90. Lee.; C.K.M.; Zhang, S.; Ng, K.K.H. Development of an industrial Internet of things suite for smart factory towards reindustrialization. Adv. Manuf. 2017, 5, 335-343. [CrossRef]

91. Civerchia, F.; Bocchino, S.; Salvadori, C.; Rossi, E.; Maggiani, L.; Petracca, M. Industrial Internet of Things monitoring solution for advanced predictive maintenance applications. J. Ind. Inf. Integr. 2017, 7, 4-12. [CrossRef]

92. Munín-Doce, A.; Díaz-Casás, V.; Trueba, P.; Ferreno-González, S.; Vilar-Montesinos, M. Industrial Internet of Things in the production environment of a Shipyard 4.0. Int. J. Adv. Manuf. Technol. 2020, 108, 47-59. [CrossRef]

93. Duhovnikov, S.; Baltaci, A.; Gera, D.; Schupke, D.A. Power Consumption Analysis of NB-IoT Technology for Low-Power Aircraft Applications. In Proceedings of the 2019 IEEE 5th World Forum on Internet of Things (WF-IoT), Limerick, Ireland, 15-18 April 2019; pp. 719-723. [CrossRef]

94. Petrov, V.; Samuylov, A.; Begishev, V.; Moltchanov, D.; Andreev, S.; Samouylov, K.; Koucheryavy, Y. Vehicle-Based Relay Assistance for Opportunistic Crowdsensing Over Narrowband IoT (NB-IoT). IEEE Internet Things J. 2018, 5, 3710-3723. [CrossRef]

95. Cao, X.; Chen, J.; Zhang, Y.; Sun, Y. Development of an integrated wireless sensor network micro-environmental monitoring system. ISA Trans. 2008, 47, 247-255. [CrossRef] [PubMed]

96. V, N.; R, L.; H, Z. Optimisation of NB-IoT deployment for smart energy distribution networks. EURASIP J. Wirel. Commun. Netw. 2019, 186, 241-255. [CrossRef]

97. Tekin, N.; Gungor, V.C. Lifetime Analysis of Error Control Schemes on Wireless Sensor Networks in Industrial Environments. In Proceedings of the 2019 27th Signal Processing and Communications Applications Conference (SIU), Sivas, Turkey, 24-26 April 2019; pp. 1-4.

98. Aftab, M.U.; Ashraf, O.; Irfan, M.; Majid, M.; Nisar, A.; Habib, M.A. A Review Study of Wireless Sensor Networks and Its Security. Commun. Netw. 2015, 7, 172-179. [CrossRef]

99. Gupta, P.; Prakash, V.; Suman, P. Noticeable key points and issues of sensor deployment for large area Wireless Sensor Network: A survey. In Proceedings of the 2016 International Conference System Modeling Advancement in Research Trends (SMART), Moradabad, India, 25-27 November 2016; pp. 164-169.

100. Khatua, P.K.; Ramachandaramurthy, V.K.; Kasinathan, P.; Yong, J.Y.; Pasupuleti, J.; Rajagopalan, A. Application and assessment of internet of things toward the sustainability of energy systems: Challenges and issues. Sustain. Cities Soc. 2020, $53,101957$. [CrossRef]

101. HaddadPajouh, H.; Dehghantanha, A.; Parizi, R.M.; Aledhari, M.; Karimipour, H. A survey on internet of things security: Requirements, challenges, and solutions. Internet Things 2019, 14, 100129. [CrossRef]

102. Rafique, W.; Zhao, X.; Yu, S.; Yaqoob, I.; Imran, M.; Dou, W. An Application Development Framework for Internet-of-Things Service Orchestration. IEEE Internet Things J. 2020, 7, 4543-4556. [CrossRef]

103. Kotsiou, V.; Papadopoulos, G.Z.; Chatzimisios, P.; Theoleyre, F. LDSF: Low-Latency Distributed Scheduling Function for Industrial Internet of Things. IEEE Internet Things J. 2020, 7, 8688-8699. [CrossRef]

104. Ma, Z.; Xiao, M.; Xiao, Y.; Pang, Z.; Poor, H.V.; Vucetic, B. High-Reliability and Low-Latency Wireless Communication for Internet of Things: Challenges, Fundamentals, and Enabling Technologies. IEEE Internet Things J. 2019, 6, 7946-7970. [CrossRef]

105. Ze, Y.; Liu, L.; Cheng, T.; Kun, Z.; Jianhua, Z. Measurement Based Characterization of Electromagnetic Noise for Industrial Internet of Things at Typical Frequency Bands. In Proceedings of the 2019 IEEE Wireless Communications and Networking Conference (WCNC), Marrakesh, Morocco, 15-18 April 2019; pp. 1-6.

106. Kumar, V.; Jha, R.K.; Jain, S. NB-IoT Security: A Survey. Wirel. Pers. Commun. 2020, 113, 2661-2708. [CrossRef]

107. Candell, R.; Kashef, M. Industrial wireless: Problem space, success considerations, technologies, and future direction. In Proceedings of the 2017 Resilience Week (RWS), Wilmington, DE, USA, 18-22 September 2017. [CrossRef]

108. Ai, Y.; Cheffena, M.; Li, Q. Radio frequency measurements and capacity analysis for industrial indoor environments. In Proceedings of the 2015 9th European Conference on Antennas and Propagation (EuCAP), Lisbon, Portugal, 13-17 April 2015; pp. 1-5.

109. Wang, H.; Mahmud, M.S.; Fang, H.; Wang, C. Wireless Health Systems. In Wireless Health; Springer International Publishing: Cham, Switzerland, 2016; pp. 9-26.

110. Varghese, B.; Buyya, R. Next generation cloud computing: New trends and research directions. Future Gener. Comput. Syst. 2018, 79, 849-861. [CrossRef]

111. Alem Čolaković, M.H. Internet of Things (IoT): A review of enabling technologies, challenges, and open research issues. Comput. Netw. 2018, 144, 17-39. [CrossRef]

112. Feng, S.; Seidel, E. Self-Organizing Networks (SON) in 3GPP Long Term Evolution; Nomor Research GmbH: Munich, Germany, 2008. 
113. NB-IoT DEPLOYMENT GUIDE to Basic Feature set Requirements. 2018. Available online: https:/ /www.gsma.com/iot/wpcontent/uploads/2018/04/NB-IoT_Deployment_Guide_v2_5Apr2018.pdf (accessed on 8 February 2021).

114. Pereira, C.; Aguiar, A. Towards efficient mobile M2M communications: Survey and open challenges. Sensors 2014, 14, 19582-19608. [CrossRef] [PubMed]

115. Babovic, Z.B.; Protic, J.; Milutinovic, V. Web performance evaluation for internet of things applications. IEEE Access 2016, 4, 6974-6992. [CrossRef]

116. Candell, R.; Papazian, P.B. Industrial Wireless Systems: Radio Propagation Measurements; National institute of Standards and Technology: Gaithersburg, MY, USA, 2017. Available online: https://nvlpubs.nist.gov/nistpubs/TechnicalNotes/NIST.TN.1951 .pdf (accessed on 8 February 2021). 